\title{
Self-Assembly and Applications of Anisotropic Nanomaterials: A Review
}

Kari Thorkelsson ${ }^{\mathrm{a} 1}$, Peter $\mathrm{Bai}^{\mathrm{a} 1}$, Ting $\mathrm{Xu}^{* \mathrm{a}, \mathrm{b}, \mathrm{c}}$

${ }^{a}$ Department of Materials Science and Engineering, University of California, Berkeley, CA 94720, USA

${ }^{\mathrm{b}}$ Department of Chemistry, University of California, Berkeley, CA 94720, USA

c Materials Sciences Division, Lawrence Berkeley National Laboratory, Berkeley, CA 94720, USA

${ }^{1}$ These authors contributed equally.

* Corresponding Author at: Department of Materials Science and Engineering, University of California, Berkeley, CA, 94720, USA. Tel: (510) 642-1632

Email address: tingxu@ berkeley.edu

Key words: Self-assembly; Nanomaterial; Nanorod; Sensing; SERS; Photovoltaics 


\section{Summary}

Anisotropic nanoparticles are ideal building blocks for a variety of functional materials due to their unique and anisotropic optical, electronic, magnetic and mechanical properties. Precise control over the orientation and spatial arrangement of these nanomaterials is often requisite to achieve coupling between nanoparticles and thereby translate the properties of individual nanoparticles to macroscopic material properties. The physics and thermodynamics involved in the self-assembly are inherently more complex than isotropic nanoparticles due to the anisotropy within the system. However, the anisotropy also introduces anisotropic nanoparticle surface chemistry and stronger interparticle interactions which could be leveraged to achieve selfassembly. To address these challenges and opportunities, a plethora of strategies have been conceived and developed to induce the self-assembly of anisotropic nanoparticles into desired nanostructures over macroscopic areas and volumes. These strategies involve manipulation of interparticle physical interactions, modification of nanoparticle surface chemistry, application of external fields, and utilization of physically or chemically patterned templates to achieve the required level of spatial and orientational control over the assembly of anisotropic nanoparticles. The resulting ordered anisotropic nanoparticle assemblies display strong plasmonic, electronic, and excitonic coupling, which render these assemblies as ideal materials for chemical and biological sensing, energy harvesting, and many other technological applications. Considering the rapid advancement in this field of research, this review aims to provide an overview of the assembly, applications, and opportunities of anisotropic nanomaterials. 


\section{Introduction}

Nanoscale materials possess many unique electrical, mechanical, and optical properties not found in bulk materials. However, precise control over the spatial distribution and orientation of these nanomaterials is often requisite to tune their ensemble properties and fully realize their use in practical applications. Macroscopic assemblies of nanoscale materials have the potential to revolutionize a wide variety of technologies, including photovoltaics, plasmonics, magnetic storage, and sensing [1-5]. The unique properties of these assembled materials may lead to significant improvements in device size and efficiency. Anisotropic nanomaterials, in particular, are increasingly important materials, as their nanoscale anisotropy in structure and composition gives rise to similarly anisotropic properties [4, 6]. However, the assembly of anisotropic nanomaterials faces additional challenges beyond those inherent in assembling isotropic particles. For some applications, such as plasmonically active materials, it is important to control the orientation of the particles relative to their neighbors or the underlying substrate [7]. In other applications, such as mechanically strengthened composites, the nanomaterial used must form an interconnected network to be effective $[6,8]$. In general, the orientation and/or connectivity of the nanoparticles relative to their neighbors must be controlled simultaneously with their spatial arrangement to harness their unique anisotropic properties.

In addition to requiring a high level of structural and orientational control, the use of ordered nanomaterials in device fabrication also necessitates a method of imposing order that is rapid, scalable, and compatible with existing processes. The "bottom-up" fabrication involves employing and manipulating particle-particle or particle-scaffold interactions to induce the assembly of the nanoscale building blocks into ordered structures, and has shown significant promise in assembling particles in a reliable and scalable manner. This class of methods is quite 
broad, incorporating approaches involving a variety of materials and techniques, including DNA [1, 9-11], functional polymers [12, 13], surface modification [14-16], external fields [17-20], and prefabricated and co-assembling templates [8, 21-24]. A recent review by Xu et al highlights the application of these aforementioned techniques on the assembly of spherical nanoparticles [25], while a recent review by Composto et al offers detailed discussion of anisotropic nanorod assembly within polymeric matrices [26]. This review aims to extend the discussion of these topics and provide a survey of the methodologies, applications, and outlook of self-assembled anisotropic nanomaterials, with an emphasis on nanorod self-assembly.

\section{Self-Assembly}

The first class of self-assembly techniques encompasses those that require neither modification of the nanomaterials nor an external field or template to drive assembly. Instead, assembly is accomplished through depletion attraction, capillary forces, dipole-dipole attractions, or some combination of these. Typically, assembly is performed in a solvent, or during solvent evaporation, to ensure the particles have enough mobility to assemble.

The depletion attraction (4-12 kT [27]), a fundamentally entropic force, comes from particles in a solvent moving close enough to each other that their excluded volumes overlap, increasing the available volume for smaller molecules or additives dissolved in the solvent [28, 29]. This results in an osmotic pressure that forces the particles together. Since the depletion attraction scales with the excluded volume overlap of the particles, particles with relatively low curvature, such as nanorods and nanocubes, experience this attraction strongly and thus assemble into well-defined structures in solution. 
Capillary forces $\left(\sim 10^{2} k T[30]\right)$, in contrast, come into effect as a solution containing particles is dried, exerting a strong force drawing parallel surfaces together as the solvent molecules between the neighboring particles evaporate [31]. Thus, as a solvent front moves through a collection of particles, it exerts forces that when carefully controlled can be used to align and pack particles.

Finally, interparticle dipole-dipole interactions (1-2 kT), i.e. the forces arising from the interaction of dipoles in two neighboring particles, can be harnessed for assembly [32]. Some materials, such as cadmium chalcogenides, possess intrinsic dipole moments (in this case an electric dipole) that can facilitate particle assembly through interaction with the dipoles on nearby particles. These dipole moments come from anisotropy in the atomic lattice of cadmium chalcogenides, the same anisotropy that makes cadmium chalcogenides useful materials for nanorod and nanotetrapod synthesis.

As far back as 1997, computational studies by Lekkerkerker et al on the phase behavior of rod-like colloids due to the depletion attraction and other pairwise attractions predicted the presence of a liquid-crystalline phase for nanorods [33], i.e. nematic assemblies forming spontaneously in concentrated nanorod solutions. This prediction was later experimentally confirmed by Alivisatos et al for CdSe nanorods [34] and Murphy et al for Au nanorods [35], and it was later shown that CdS nanorods exhibit self-assembly into bundles at far lower concentrations than previously observed [36]. The depletion forces between nanoparticles leading to this assembly can be precisely controlled through the amount of hydrocarbon additives to the nanoparticle solution, allowing assembly of not only nanorods [28], but also tetrapods and octapods [37] (Fig. 1). In this way, micrometer-sized sheets of aligned anisotropic nanoparticles could be constructed. 

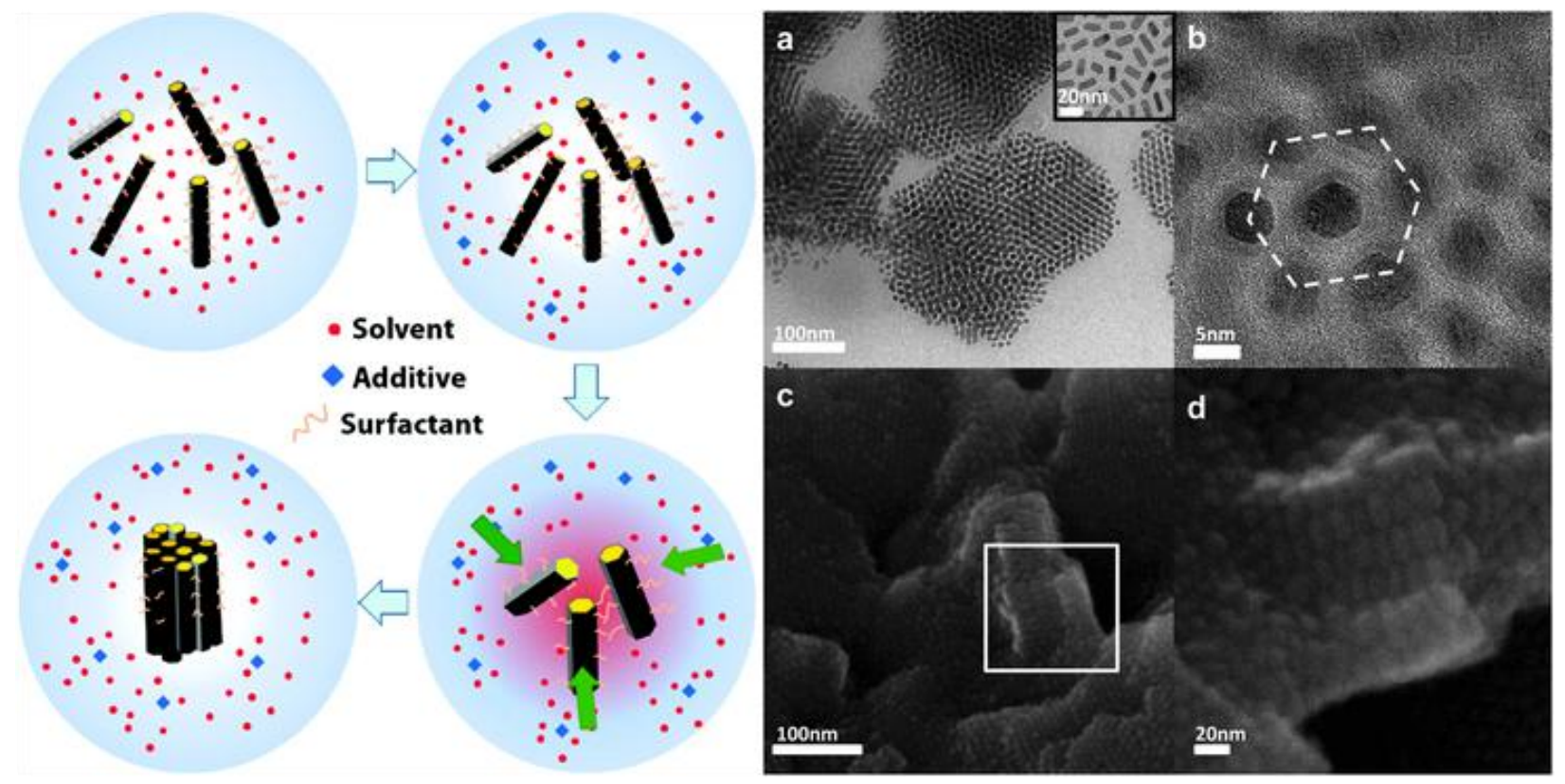

Figure 1: Assembly using the depletion attraction. Left: schematic showing depletion attraction due to exclusion of additive. Reprinted with permission from [28]. Copyright 2010 American Chemical Society. Right: TEM and SEM of assemblies formed. a) TEM image of assembled $\mathrm{Fe}_{2} \mathrm{P}$ nanorods. Inset: nanorods before assembly b) High magnification view showing hexagonal packing symmetry. c) SEM image of multilayer bundles. d) Magnified view of boxed area in c). Reprinted with permission from [37]. Copyright 2010 Royal Society of Chemistry.

Capillary forces have also been effective in inducing nanorod assembly. Specifically, capillary forces between adjacent nanorods become significant as solvent within a nanorod solution evaporates. Earlier approaches found success in allowing the solvent edge to move from one side of the film to another by suspending a substrate at the surface of a solution and allowing the surface of the solution to move over the substrate as the solvent evaporated. In this manner, gold nanorods were assembled on TEM grids [38] by Alivisatos et al, and CdSe nanorods were assembled on a mica substrate [39] by El-Sayed et al. Although crystalline structures were formed in both cases, this approach also demonstrated the formation of phases with little positional order. For example, the CdSe nanorod assemblies formed phases ordered around a central vortex-like structure. In either case, the convective flow of the solvent across the substrate left the rods parallel to the surface. Placing drops of the solution onto a substrate and allowing them to 
evaporate in a method known as drop casting showed similar results: cadmium chalcogenide nanorods self-assembled into stripe patterns when drop cast due to a combination of capillary forces and interparticle forces arising from the dipole moment of the nanorods [40].

Expanding on this research, concentration dependence was examined - using a smaller amount of nanorods results in the formation of characteristic ring-like 'breath figures' on the substrate [41]. Shape dependence was investigated - assembling particles in solutions containing mixtures of rods and spheres results in the gold nanorods demonstrating a self-selective behavior, assembling into bundles excluding the spheres [42]. The effect of the drying kinetics of nanoparticle solutions was probed [43] and similar principles were eventually applied to generate device-scale perpendicular alignment of CdS nanorods [44], as shown in Figure 2.
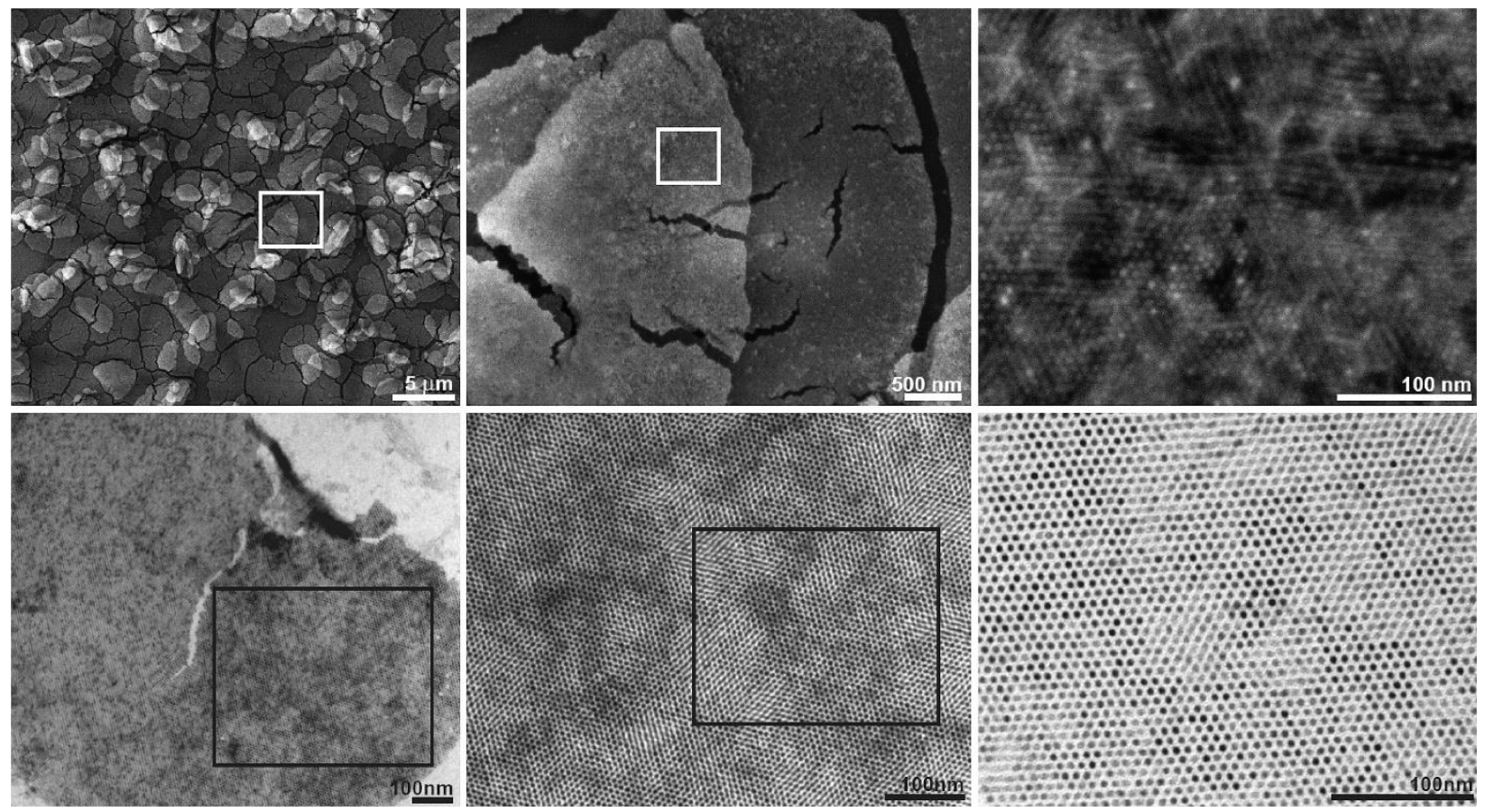

Figure 2: SEM (top row) and TEM (bottom row) of large vertically oriented nanorod domains produced through solution casting. Each image shows higher detail of the boxed area in the image to its left. Reprinted with permission from [44]. Copyright 2010 American Chemical Society. 
As the library of achievable nanocrystal shapes has expanded with time, so have efforts to assemble them. It has been shown in simulations that under the right conditions, nematic phases can be formed by tetrapods and cubatic phases can be formed by both tetrapods and cuboidal particles $[45,46]$. Likewise, simulation has also been used to show that a variety of convex space-filling polyhedra can be assembled through entropic forces into crystalline, liquidcrystalline and plastic-crystalline phases, which are phases with some orientational or conformational degrees of freedom $[47,48]$. Experimentally, triangular prisms of $\mathrm{LaF}_{3}$, when combined with spherical particles, form well-defined superlattices [49], and when confined to a surface, tessellate across the surface [50]. Octapods confined to a monolayer can also be assembled into well-defined square, rhombic, or hexagonal lattices [51, 52], or chains or threedimensional lattices when unconfined [53]. A number of different shapes of gold nanostructures, including nanorods, nanocubes, and other polyhedra, can be assembled into close-packed lattices upon solvent evaporation [54]. Silver polyhedra have been similarly assembled [55]. Rhombic dodecahedra formed by MOF crystals have also been successfully assembled into superlattices in this manner [56]. Nanoplates represent another class of nanoscale building blocks which selfassemble into superlattices and liquid crystalline mesophases upon solvent evaporation. The detailed description of their assembly and properties are more comprehensively reviewed elsewhere [57-59].

Dipole-dipole interactions have also been used to drive assembly, as some nanorods have dipoles that are strong enough to cause assembly into superlattices directly. The strong magnetic dipole found in ferromagnetic cobalt nanorods, for example, allows for their simple assembly into superlattices [60]. 
Finally, it is also possible to manipulate the solution in which the particles are suspended to control interparticle forces and therefore assembly. For example, Weller et al showed that optically anisotropic 3D superstructures can be formed from $\mathrm{CdSe}$ and $\mathrm{CdS}$ nanorods by allowing a nonsolvent to diffuse into the nanocrystal solution [61]. The slow introduction of nonsolvent caused the nanorods to cluster together into bundles of aligned rods in a manner reminiscent of the depletion attraction-driven assemblies above.

This research has shown that a variety of unmodified anisotropic nanostructures have a tendency to self-assemble, given the right conditions. By taking advantage of depletion attractions, dipole-dipole attractions, or capillary forces, researchers have achieved aligned nanoparticle superlattices, some of macroscopic size. These methods provide a number of useful candidates for the self-assembly of anisotropic nanomaterials into macroscopically sized closepacked lattices.

\section{Directed Assembly via Surface Modification}

Another approach to nanoparticle assembly involves modifying the nanoparticle surface, typically through ligating functional molecules to the surface or through the modification of existing ligands. This approach can generate a variety of structures unique from solvent evaporation methods, including end-to-end chains and networks of nanorods, and also tends to function well over large nanorod concentration ranges.

One method relying on this approach involves the conjugation of DNA strands to the particle surfaces. For example, by binding non-complementary strands of DNA to the particles, then inducing assembly by adding a strand half complementary to each, Wang et al assembled gold nanorods into clusters exhibiting a characteristic interparticle spacing corresponding to the 
length of hybridized DNA used [10]. This approach was later extended by Mirkin et al to the formation of different nanoparticle superlattices through surface functionalization of anisotropic particles - including nanorods, triangular prisms, rhombic dodecahedra, and octahedra - with DNA [11]. Some of these assemblies are shown in Figure 3. This method has continued to stimulate both computational and experimental research interest $[62,63]$.

a

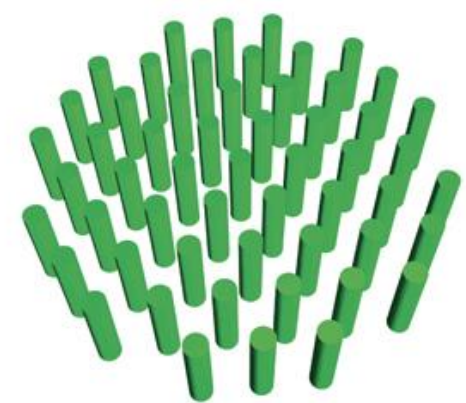

b

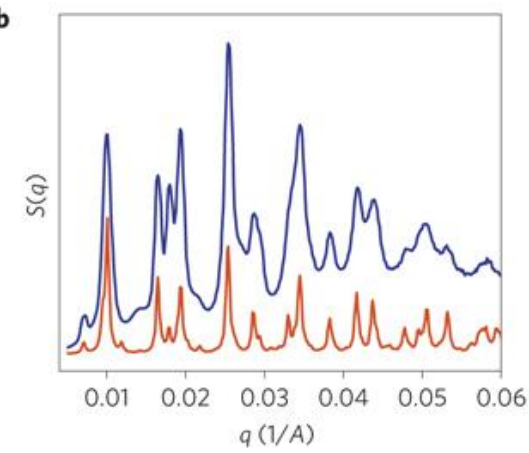

c

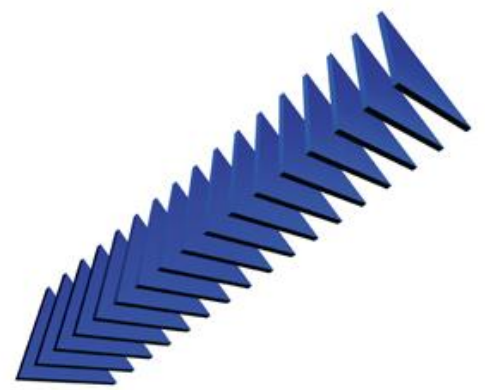

d

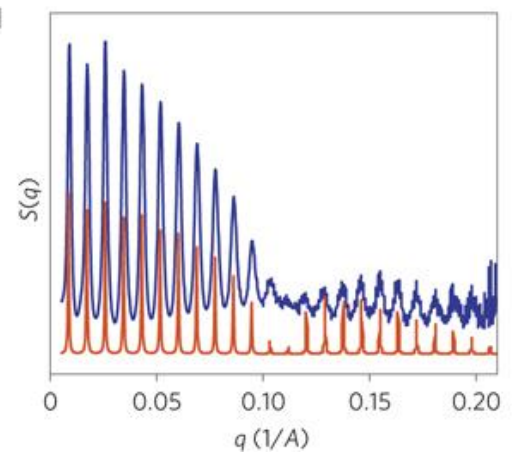

e
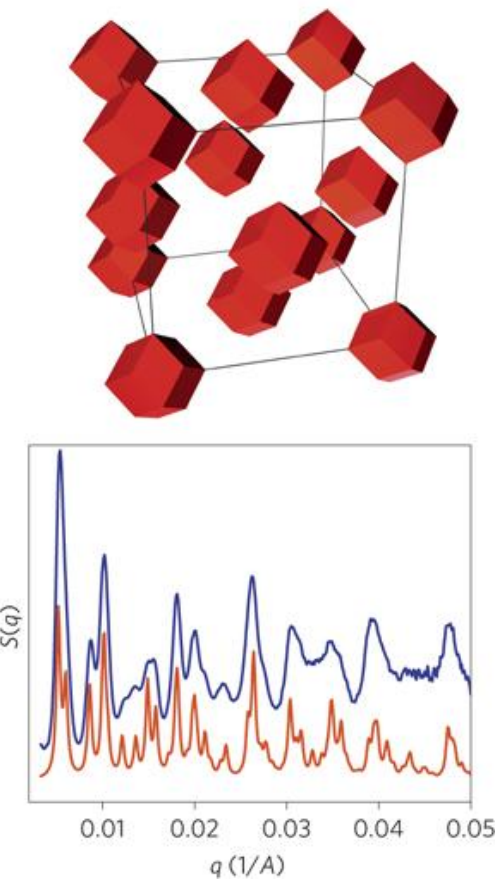

Figure 3: SAXS characterization of DNA-guided nanoparticle assemblies. a) Schematic of one layer of a hexagonally packed assembly of gold nanorods. b) Experimental (blue) and simulated (red) SAXS pattern of gold nanorods assembled as in a). c) Schematic of 1D lamellar assembly of gold triangular nanoprisms. d) Experimental (blue) and simulated (red) SAXS pattern of assembly illustrated in c). e) Schematic of 3D FCC assemblies of gold rhombic dodecahedra. f) Experimental (blue) and simulated (red) SAXS patterns of assembly illustrated in e). Reprinted with permission from [11]. Copyright 2010 Nature Publishing Group.

Besides DNA, other types of molecules have been used as ligands to guide nanorod assembly. Several studies used cysteine, glutathione and dithiol ligands end grafted to nanorods to achieve tunable and reversible end-to-end nanorod assembly [64, 65]. Another ligand that has been used is a carboxylic acid derivative with a thiol moiety that binds gold nanorods together using thiol-gold bonds and hydrogen bonds [66]. The particles assembled primarily end-to-end, 
demonstrating that gold nanoparticles tend to be more chemically active at the ends, likely due to reduced ligand coverage. This difference in chemical activity was later leveraged by Kumacheva et al to functionalize the end and side facets with different molecules, after which varying the solvent conditions would induce the formation of bundles, chains, disks, and hollow spheres [12], as shown in Figure 4. This preferential bonding and the ability to control interparticle forces through it allows the use of such anisotropic particles as "colloidal molecules," enabling the formation of complex structures, including, for example, diamond structures [67].

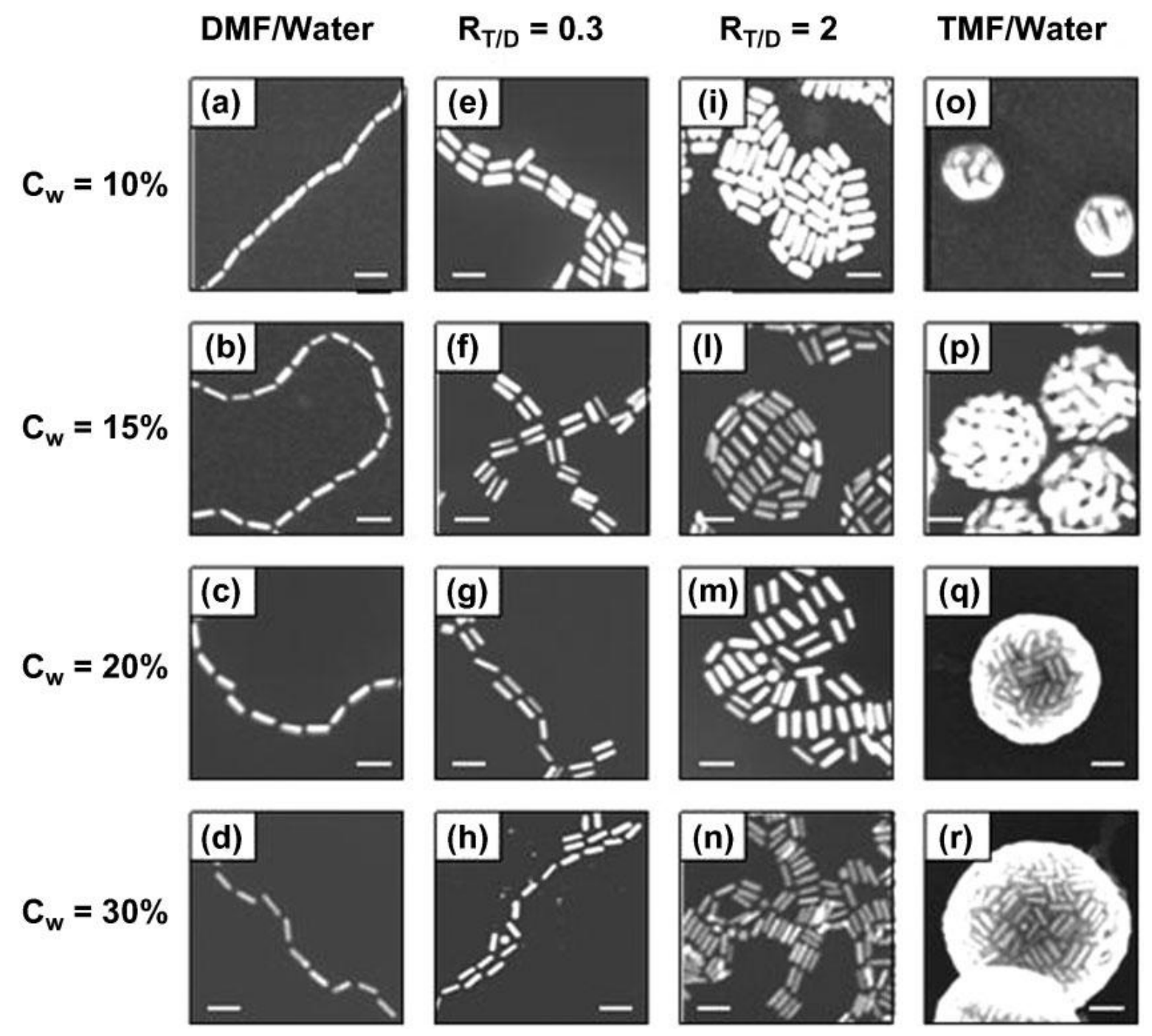


Figure 4: SEM images of gold nanorods with different end- and side-functionalization as a function of water content $\left(\mathrm{C}_{\mathrm{w}}\right)$ and ratio of THF to DMF $\left(\mathrm{R}_{\mathrm{T} / \mathrm{D}}\right)$. When in a selective solvent for the sides of the nanorods (DMF, left column), the nanorod ends aggregated, forming chains. When in a poor solvent (THF, right column), the nanorods aggregated into spherical clusters. Reprinted with permission from [12]. Copyright 2008 Wiley.

Similar methods have been investigated as well, primarily involving end-to-end assembly of nanorods. Linking functionalized gold nanorods end-to-end has been accomplished using, among others, biotin functionalization in the presence of streptavidin [68-70]; terpyridine functionalization in the presence of iron ions [71]; and a polypeptide that aggregates at low $\mathrm{pH}$, allowing reversible assembly [16]. Other methods include using a polymer with a lower critical solution temperature bound onto the sides of nanorods to reversibly induce assembly either through heating or through irradiation with a wavelength absorbed by the particles [72]. However, not all of these assembly methods require leveraging side-selective chemistry. Nanorods have also been assembled both end-to-end and side-to-side at once to form lattices using a dimercaptosuccinic acid [73].

Other versatile assembly methods allow selective assembly either end-to-end or side-toside according to the environment. Such behavior has been enabled by Lavrentovich et al with a lyotropic chromonic molecule [74], which switches between the types of assembly upon tuning the gold nanorod surface charge. A similar approach using phospholipid ligands has also been employed by Voigt et al [75], enabling switching between end-to-end and side-to-side assembly by adjusting the electrostatic interactions between nanorods.

In a similar vein, other shapes of particles can be assembled as well. Xia et al assembled silver nanocubes by selectively functionalizing some number of the cube faces [14]. By functionalizing one face, dimers were formed; functionalizing two faces resulted in chains, and so on up to a close-packed superlattice. The synthesis of cadmium chalcogenide particles with 
gold tips has provided a new avenue of selectively manipulating the ends of the rods due to the different chemical affinity of the ends and sides of the rods. Binding different ligands to the gold and the $\mathrm{CdSe}$ in these rods and adjusting the solvent quality allows for the formation of closepacked superlattices [76], mirroring methods that take advantage of differing chemical activity at different sites on a particle. Functionalizing the gold tips with Biotin-disulfide resulted in the formation of nano-flowers using Avidin as a linker molecule [77]. The chemical structures of these particles also allow for an alternate approach to assembling this type of nanorod: by destabilizing the gold tips using iodine, the tips of separate particles can be fused together into larger gold domains [78], which unite the rods end-to-end.

Larger molecules can also be attached to the nanoparticles to facilitate assembly. A number of simulations have been performed to investigate the behavior of nanorods with a single large oligomeric tether. These studies demonstrate that various self-assembled structures can form depending on ligand length, rod length, rod aspect ratio, and the presence of solvent [13, 79-82], including nematic phases, multilayer smectic arrowhead phases, lamellae, among others.

\section{External Field-Directed Assembly}

Still another approach to nanoparticle assembly is the application of external force, typically via an electric, magnetic, or shear field, to restrict the particle location. These methods, while requiring the addition of forces external to the nanoparticles, can often achieve high levels of alignment in a straightforward manner.

The most commonly used external field is the electric field, which can be used to polarize nanoparticles, causing them to form chains or colloidal crystals. It is typically applied by setting a high voltage across a pair of planar electrodes on either side of a slowly drying solution. This 
type of assembly has been demonstrated by Velev et al with particle compositions ranging from metallic [83] to dielectric [84]. Anisotropic nanoparticles possessing a dipole moment, such as CdS or CdSe nanorods, can be particularly well organized through the application of a DC electric field, as there is no need to polarize the particle. Assembly of nanorods in this manner has been investigated both experimentally as well as computationally [18, 32]. By using UV light to form highly polarizable excitons and increase the dipole moment of CdSe nanorods, this driving force for alignment was further increased [85]. Other variants of electric field-directed assembly employ a matrix other than a solvent: by including a polymer immiscible with $\mathrm{CdSe}$ nanorods, the nanorods were induced to form superlattices as they macrophase-separated from the polymer [86] as shown in Figure 5.
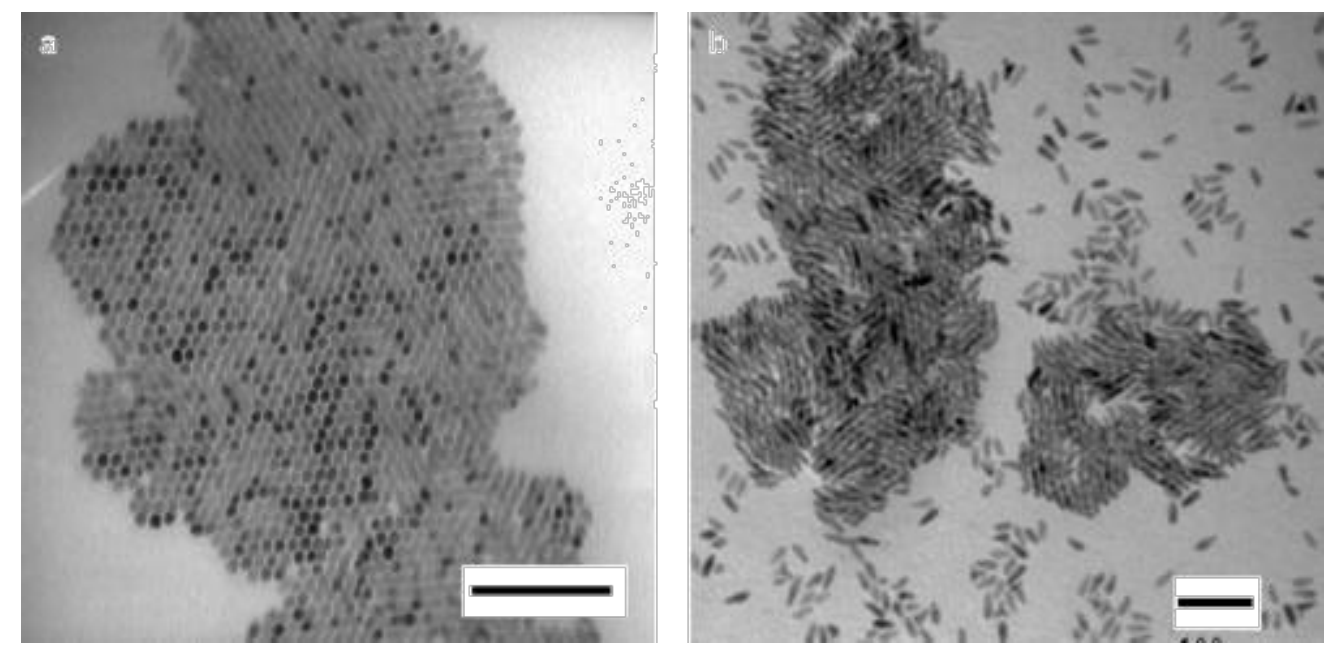

Figure 5: TEM images of nanorod superlattices formed by "self-corralling" behavior in the presence of a polymer a) with and b) without an applied electric field. Scale bars are $100 \mathrm{~nm}$. Reprinted with permission from [86]. Copyright 2006 American Chemical Society.

Some nanomaterials also have a magnetic moment that can be used to align them. While ferromagnetism does not occur in small $(\mathrm{d}<20 \mathrm{~nm})$ particles, some nanomaterials that exist on this size scale can still be manipulated with a magnetic field due to the magnetic susceptibility of the material. Carbon nanotubes, for example, have been so aligned by Smalley et al [19]. 
However, most research on this topic has been toward the organization of physically isotropic (though in some cases magnetically anisotropic) particles [87], likely due to the permanent magnetic dipole present in many physically anisotropic nanoparticles that causes spontaneous self-assembly without requiring an external field [60].

However, not all types of nanorods are sufficiently susceptible to electric or magnetic fields to enable alignment in this manner. For such particles, an alternate method that has seen use is the application of a shear field, typically applied by deforming a matrix containing the particles. Nanorods have been aligned in polymer films by stretching the films, resulting in alignment to within nine degrees of the applied tension [17]. In a slightly different approach, a direct solution drawing method has been used to apply shear to gold nanorods within a polymer fiber as shown in Figure 6, also resulting in good alignment [20]. By embedding aligned gold nanorods within an electrospun fiber, it was demonstrated that the plasmon shifts due to nanorod proximity could be used to measure swelling of the fibers [88]. 


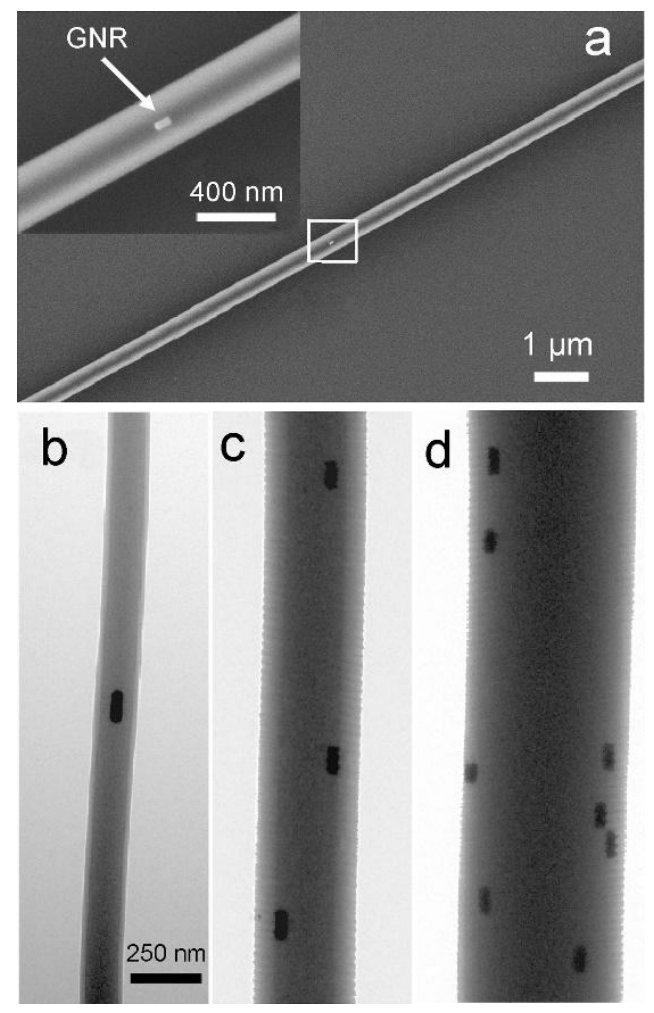

Figure 6: a) SEM image of gold nanorods embedded in a polyacrylamide fiber. Inset: higher magnification detail of nanorod near surface. b-d) TEM images of gold nanorods in fibers with diameters 150,360 , and $600 \mathrm{~nm}$, respectively. Scale bar in b) applies to c) and d) as well. Reprinted with permission from [20]. Copyright 2012 American Chemical Society.

It is also possible to suspend particles that are not susceptible to an external field in a matrix that responds to such a field. Gold nanorods in a nematic liquid crystalline fluid, for example, allow for alignment by using a magnetic field $[7,89]$ or shear field $[90,91]$ to align the liquid crystalline molecules, which subsequently forces the nanorods into alignment. Moreover, the liquid crystalline matrix itself can be considered as an external field as anisotropic nanoparticles have been demonstrated to assemble into nematic [90-94] and cholesteric [93, 94] phases without the application of additional external fields.

\section{Templated Self-assembly}

Finally, assembly of anisotropic nanoparticles can be achieved by using a template to impose order on the particles. The simplest possible template is a surface or interface at which to 
assemble particles. Well-aligned layers of nanorods can be assembled using a Langmuir-Blodgett monolayer [95] as shown in Figure 7, or on the surface of organic solvent droplets suspended in water [96]. The same principles have been applied to assemble nano-tetrapods at an air-water interface, forming ribbons that upon an increase in surface pressure collapse into interconnected networks [97]. More highly curved interfaces have been used as well. The surface of multi-wall carbon nanotubes has been used to assemble gold nanorods, and due to the high curvature of the nanotubes, this had the effect of aligning the nanorods parallel to the axis of the nanotubes [98].
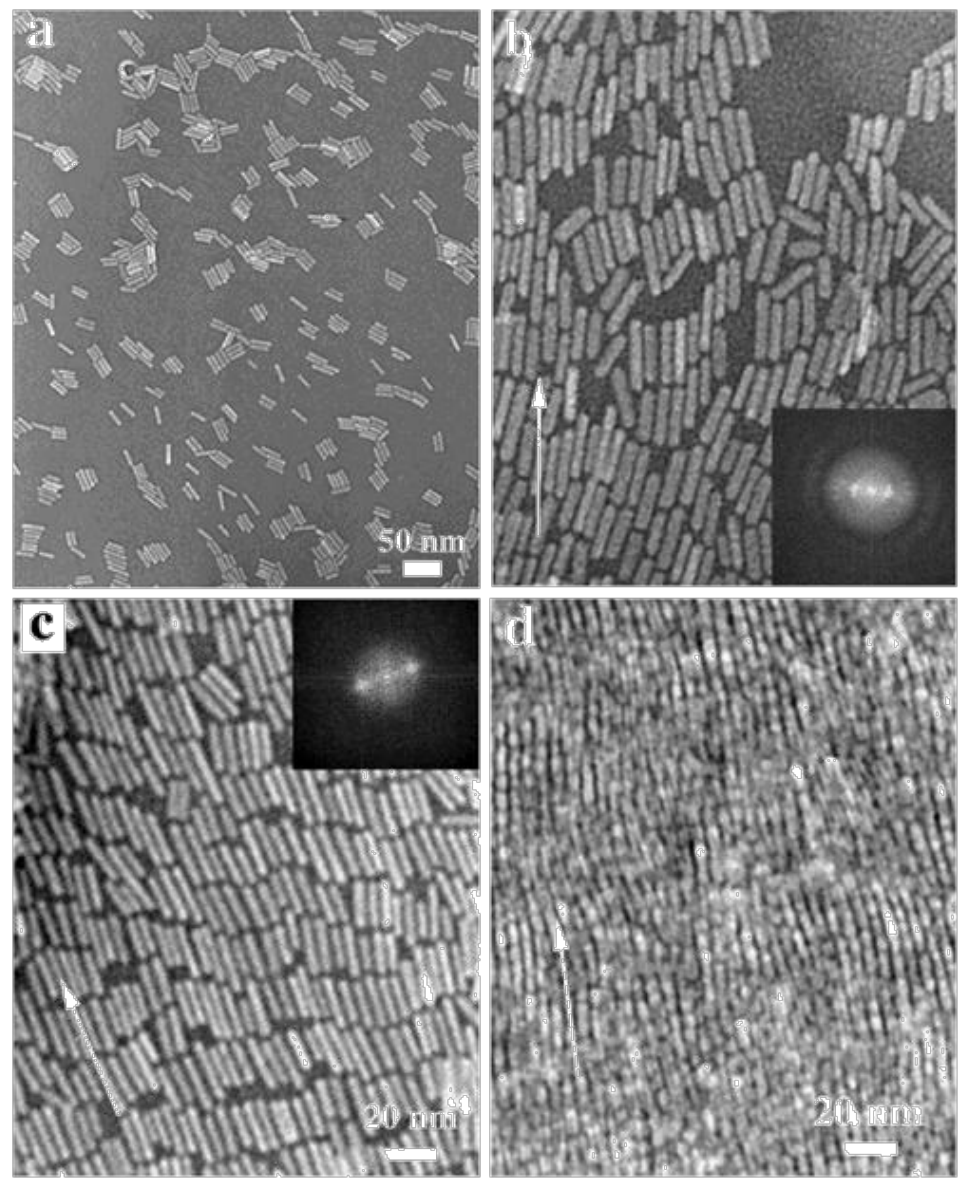

Figure 7: TEM images of nanorod assemblies at water/air interface with increasing compression. a) isotropic distribution at low pressure; b) monolayer with partial nematic arrangement; c) monolayer with smectic arrangement; and d) nanorod multilayer with nematic configuration. Insets in panels b) and c) are the Fourier transform of the corresponding image. Reprinted with permission from [95]. Copyright 2001 American Chemical Society. 
A patterned substrate can be used to align and distribute nanoparticles more precisely than a flat surface. For example, trenches etched into a substrate can align nanotetrapods deposited from a solvent $[99,100]$. By controlling the width of the trenches, two of the tetrapod arms can be aligned with the longitudinal direction of the trench.

A chemically patterned substrate can also be used. Vaia et al formed arrays of nanorods using a substrate patterned with alternating stripes of polystyrene and poly(2-vinylpyridine) [101]. The rods preferentially deposit on the polyvinylpyridine stripes, allowing for fine control over their position. By controlling the width of the stripes, the nanorod orientation can be controlled.

Yet another way to externally manipulate nanoparticle arrangement involves the use of polymers as a ligand or a matrix. Upon dispersal in a polymer film, gold nanorods demonstrate local ordering [22] with adjustable levels of aggregation controlled by the relative lengths of the polymer chains in the matrix $(\mathrm{P})$ and in the ligand brush layer on the nanorods $(\mathrm{N})$ [102]. Aggregation was observed for $\mathrm{P} / \mathrm{N}>2$ for nanorods with an aspect ratio of 3.3. Further studies expanded this to a larger range of aspect ratios and explored the energetic contributions controlling the assembly, showing that the assembly was almost entirely due to depletion attractions and that interparticle spacing in the aggregates was determined solely by the degree of polymerization of the nanorod ligand brush [103]. Some examples of this assembly can be seen in Figure 8. 

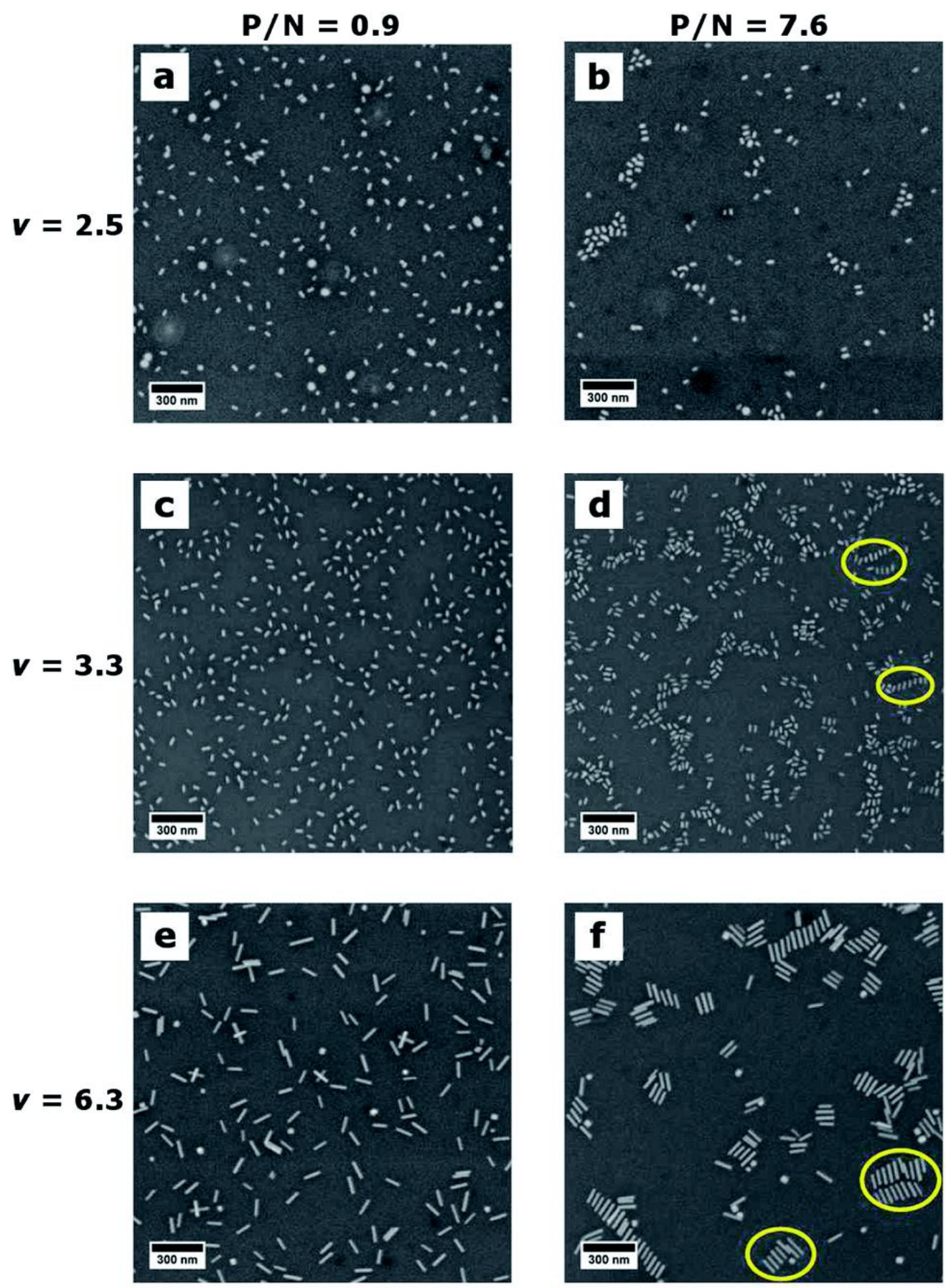

Figure 8: SEM images of PS-Au NRs (N) in PS (P) films as a function of aspect ratio, $v=(a$ and b) 2.5, (c and d) 3.3, and (e and f) 6.3. The left column shows the dispersion of PS-Au NRs for $\mathrm{P} / \mathrm{N}=0.9$. The right column shows that the PS-Au NRs assemble into clusters for $\mathrm{P} / \mathrm{N}=7.6$. The yellow circles denote well-ordered columns of side-by-side nanorods. The nanorod volume fraction is approximately 5\%. Scale bars are $300 \mathrm{~nm}$. Reprinted with permission from [103]. Copyright 2014 Royal Society of Chemistry. 
Blends of polymers can increase the complexity of the system and allow for the formation of co-continuous microstructures. Through simulation, Balazs et al showed that incorporating nanorods into one phase of a phase-separating blend yielded a percolating network of nanorods within the continuous minority phase [8]. This morphology results in a significantly stiffer and more electrically conductive composite compared to one in which the nanorods are randomly distributed throughout the composite. A similar morphology was later produced through the inclusion of a large amount of nanorods in one phase of a phase-separating polymer blend, leading to kinetic arrest of a co-continuous morphology through jamming of the nanorods [23]. The aggregation of the nanoparticles results in a significant decrease in the size of the polymer domains upon demixing, and the presence of the nanorods prevents these small domains from coalescing into larger ones.

More complex anisotropic particles in polymer blends have also been explored. Janus nanorods, which are both physically and chemically anisotropic, were examined in polymer blends through simulations by Yan et al [104]. Incorporation of the nanorods led to a decrease in the assembly kinetics of the system, stabilizing ordered hierarchical structures that formed during shear flow of the composite, in turn leading to macroscopic alignment of the nanorods.

Using a structured polymer matrix instead of a homopolymer allows for the incorporation of a template into the assembly process. BCPs self-assemble into a variety of structures, including spheres, cylinders, lamellae, and bicontinuous phases for a diblock copolymer - and rapidly growing more complex for triblock copolymers [105]. When a single block interacts preferentially with the nanoparticles or their ligands, nanoparticles will assemble within the block, allowing the morphology of that block to template the nanoparticle assembly. 
BCP-templated systems have received a good deal of attention and analysis, both computational and experimental. Simulations by Ma et al showed that BCP microdomains could be used to order nanorods into nanowire structures within the microdomains [106]. This type of assembly has proved to be more complex than other types of templated assemblies as the $\mathrm{BCP}$ morphology is not only influenced by the polymer used, but also the size and aspect ratio of the nanorods co-assembled within [107-110]. However, mirroring similar simulations on nanorods in polymer mixtures, improvements in Young's modulus and composite strength were observed. The important factor in this strengthening process proved to be the aspect ratio of the nanorods. Nanorod location - whether within a domain or at the interface between microdomains - had little effect [111]. By controlling the organization of the BCP template through external forces, macroscopic order could be imparted to the nanorods through this type of assembly [112].

Other simulations have focused on other forms of anisotropic particles. Clay-BCP composites, in particular, have received significant attention as a potentially useful route for mechanically strengthening polymer composites [113]. The clay forms platelets nanometers in thickness within one of the polymer domains, resulting in a significant strengthening in the mechanical properties of the BCP. Other, more esoteric anisotropic materials, such as chemically anisotropic Janus particles, have been explored as well. Since a BCP has at least two chemically distinct domains, the surface of the Janus particles can be tuned to adjust their location and orientation within the BCP matrix, allowing for the potential production of oriented macroscale structures through the imposition of an external force to induce macroscopic ordering of the $\mathrm{BCP}$ [114].

Due to the effective intercalation of $\mathrm{BCP}$ between clay sheets, clay-BCP composites have been extensively investigated experimentally [115-117]. This intercalation allows for easy 
separation of the clay into thin, nanometer-thick sheets within the composite, reducing its effect on the optical properties of the polymer while maintaining a large amount of the strength of the clay. More recently, the co-assembly of BCPs with one-dimensional nanomaterials has also received significant experimental attention. In one study, Composto et al demonstrated that gold nanorods were aligned parallel to a substrate supporting the BCP-Au NR film due to the confinement of the nanorods by the lamellae of the BCP [21]. The results of this assembly can be seen in Figure 9. Though the nanorods are oriented randomly within the plane of the lamellae (Fig. Error! Reference source not found.c), $71 \%$ of the nanorods are restricted to within $5^{\circ}$ of this lamellar plane. A similar approach has been used by Zhu et al to form 1D assemblies of nanorods by confining them within a BCP cylinder, yielding either end-to-end or twisted side-toside chains of rods encapsulated within wormlike BCP micelles [118]. Alternately, Shenhar et al demonstrated that the surface of a BCP thin film can provide two chemically distinct surfaces suitable for assembly [24]. The nanorods assemble on the surface, forming hierarchical smecticlike assemblies on one microdomain while avoiding the other. 

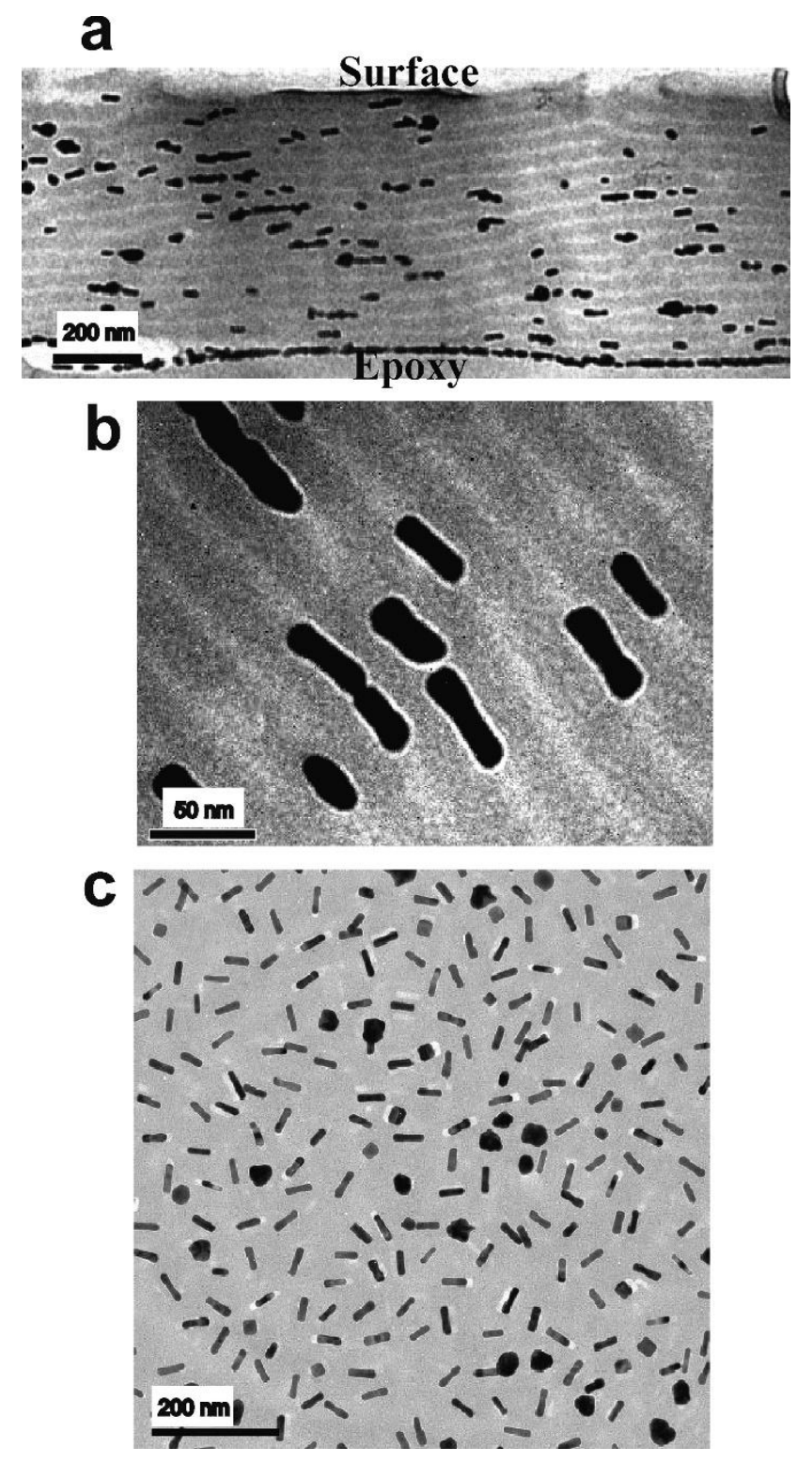

Figure 9: a) Cross-sectional TEM image of gold nanorods in a polystyrene(PS)-blockpolymethylmethacrylate (PMMA) film. Dark and light stripes represent PS and PMMA, respectively. b) Higher magnification TEM image of the nanocomposite film showing nanorods oriented within PMMA lamellae. c) Top-down view of film. Reprinted with permission from [21]. Copyright 2007 American Chemical Society.

Though BCPs are useful for some aspects of anisotropic nanomaterial assembly, they are not without shortcomings. Particles that are smaller than the blocks used are not well-confined by the $\mathrm{BCP}$, and can distribute themselves throughout that microdomain. Some improvements on this system have been offered, in particular the use of a BCP-based supramolecular system to 
confine nanorods between rigid comb-like blocks. Xu et al have demonstrated that nanorods of a variety of chemistries can be assembled within a supramolecular nanocomposite in bulk. The assembly can be tailored such that two highly sought after morphologies, nanoscopic networks of nanorods and nanorod arrays parallel to the cylindrical $\mathrm{BCP}$ microdomains, can be obtained by tuning the rod-rod and rod-polymer interactions [119] (Figure 10a-c). Similarly, nanorods with aspect ratios from 8-12 can be aligned in an end-to-end fashion in cylindrical supramolecule thin films by achieving a balance in rod-rod and rod-polymer enthalpic interaction, polymer chain conformational entropy, and nanorod translational and orientational entropy (Figure 10d-f) [120]. Recent advances in BCP and BCP supramolecule directed nanorod assembly are also discussed extensively in a review [25]. 

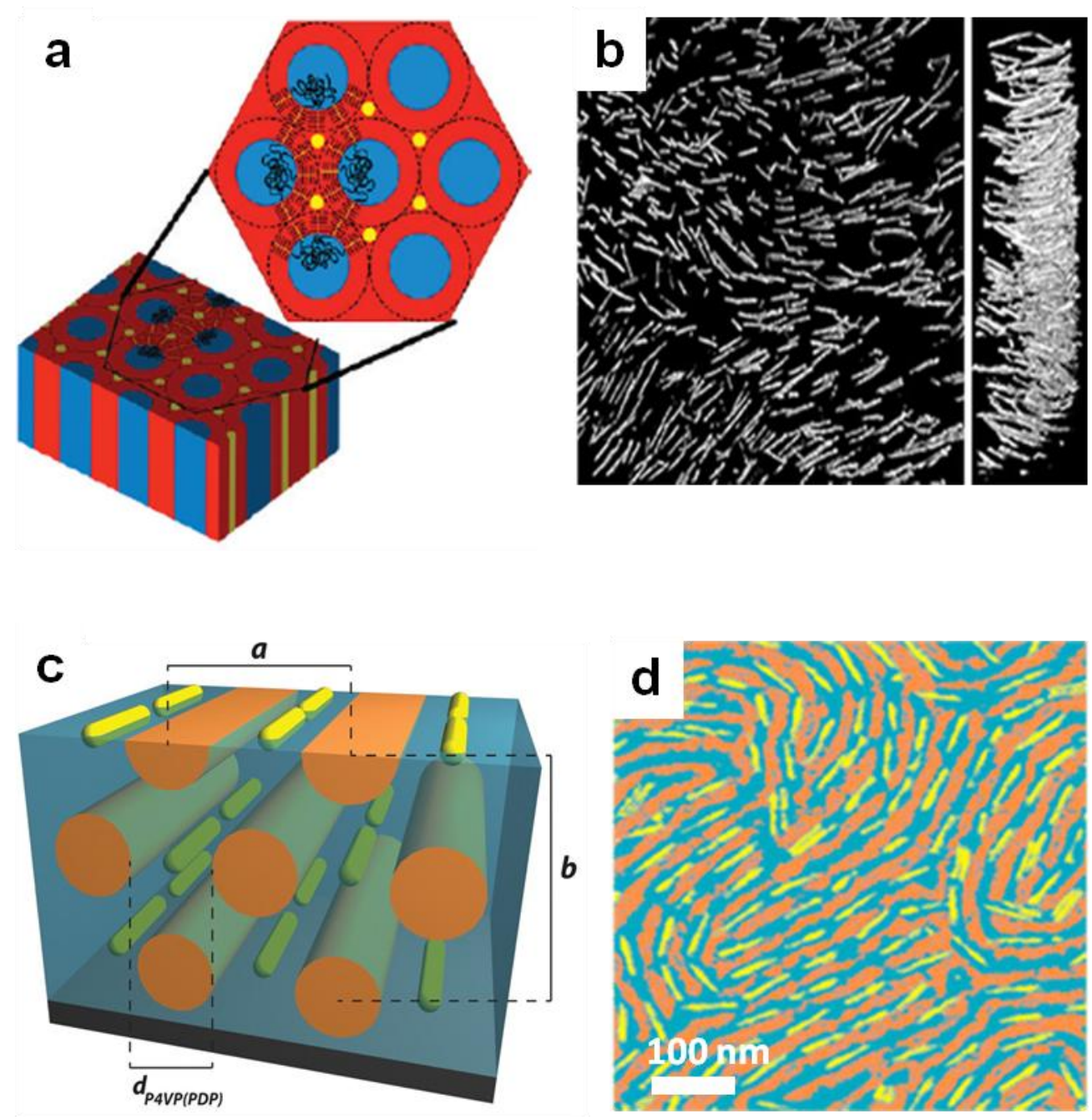

Figure 10: Nanorod assembly within BCP-based supramolecule. a) Schematic drawing of CdS nanorods (yellow) assembled in a cylindrical supramolecule. b) 3-D TEM tomography reconstruction showing inplane (left) and cross-sectional (right) views of nanorods assembled within the cylindrical supramolecule. Reprinted with permission from [119]. Copyright 2012 American Chemical Society. c) schematic showing thin film assembly of CdS nanorods (yellow) in poly(4-vinylpyridine)(3-pentadecylphenol) supramolecular block (teal) among polystyrene cylinders (orange). d) AFM phase image of nanocomposite film demonstrating assembly. The colors of the nanorods and supramolecule are modified to match the schematic shown in c). Reprinted with permission from [120]. Copyright 2013 American Chemical Society.

\section{Applications}

The intriguing properties of anisotropic nanomaterials enable a wide variety of potential applications including sensing, energy harvesting, and energy transport, to name a few. 
Individual nanorods already possess some unique attributes to be utilized in certain applications, and these studies are reviewed extensively elsewhere [121-123]. However, to harness the full potential of nanorods, many applications require specific assemblies of nanorods so the properties of individual nanorods can couple, or be triggered simultaneously, and translate into desired macroscopic properties. Plasmonic coupling of assembled metallic nanorods lead to highly precise sensors with low limits of detection, and coupling of tunable optical and electronic properties in assembled semiconducting nanorods have potential in photovoltaics and energy transport.

\section{Assembly-triggered Sensing}

In both bulk and nanoscale metallic materials, electrons in the conduction band oscillate upon excitation from incident electromagnetic wave irradiation, leading to a phenomenon known as localized surface plasmon resonance (LSPR). This oscillation induces charge separation between the free electrons and the metal core, which in effect results in electric dipole oscillations. In anisotropic nanomaterials, LSPR has different peaks due to oscillations longitudinal and transverse to the long axis of the nanorod. The peak positions and intensities depend on the composition, size, and aspect ratio of individual nanorods. Specifically, the transverse peak is in the visible wavelength regime $(\sim 500 \mathrm{~nm})$ and is insensitive to nanorod size, while the longitudinal peak can be significantly red-shifted with increasing nanorod aspect ratio [124, 125], as predicted by the Gans Theory [126]. In addition, the presence of other nanorods in proximity can cause coupling of electronic oscillations between adjacent nanorods, which also affects LSPR peak position and intensity [127-130]. These phenomena are often utilized to great effect in nanorod assemblies for chemical and biological sensing. 
In some cases, the target analyte is used to trigger the assembly or disassembly of nanorods, which leads to changes in the inter-rod LSPR coupling, and ultimately changes in LSPR peak shape [131], position [131-134], intensity [135-139], and ratio of intensities in the longitudinal and transverse LSPR peaks [140].

End and side functionalization of nanorods is frequently used to trigger the desired assembly response in the target analyte solution. Merkoci et al demonstrated that Au nanorods functionalized with an $\mathrm{N}$-alkylaminopyrazole ligand was able to coordinate heavy metal ions such as $\mathrm{Zn}^{2+}, \mathrm{Cd}^{2+}, \mathrm{Hg}^{2+}, \mathrm{Cu}^{2+}, \mathrm{Pb}^{2+}$ and $\mathrm{As}^{3+}$, leading to nanorod assembly into 1-D chains and resulting in red-shift and broadening of the longitudinal plasmon band under as low as $3 \mathrm{ppt} \mathrm{Hg}^{2+}$ exposure and reduction in the plasmon band intensity for other ions [131]. Au nanorods modified with 11-mercaptoundecanoic acid (MUA) formed end-to-end assemblies in the presence of $\mathrm{Eu}^{3+}$ and lysine due to coordination binding between $\mathrm{Eu}^{3+}$ and $\mathrm{COO}^{-}$group of the MUA, and electrostatic interaction of the $\mathrm{COO}^{-}$group with $\mathrm{NH}^{3+}$ of lysine. The resulting red shift of the longitudinal LSPR is effective in detecting lysine concentrations as low as $1.6 \times 10^{-6} \mathrm{M}$ [133] (Figure 11). Au nanorods with cetyltrimethylammonium bromide (CTAB) formed end-to-end and eventually side-to-side assemblies under increasing concentrations of ethylenediaminetetraacetic acid (EDTA) due to chelation, resulting in a red shift of the longitudinal LSPR. However, upon addition of stronger chelating agents such as $\mathrm{Pb}^{2+}$, EDTA detached from the nanorods and formed stronger complexes with $\mathrm{Pb}^{2+}$, resulting in disassembly of the nanorod superstructures and blue shift of the transverse LSPR [134]. 

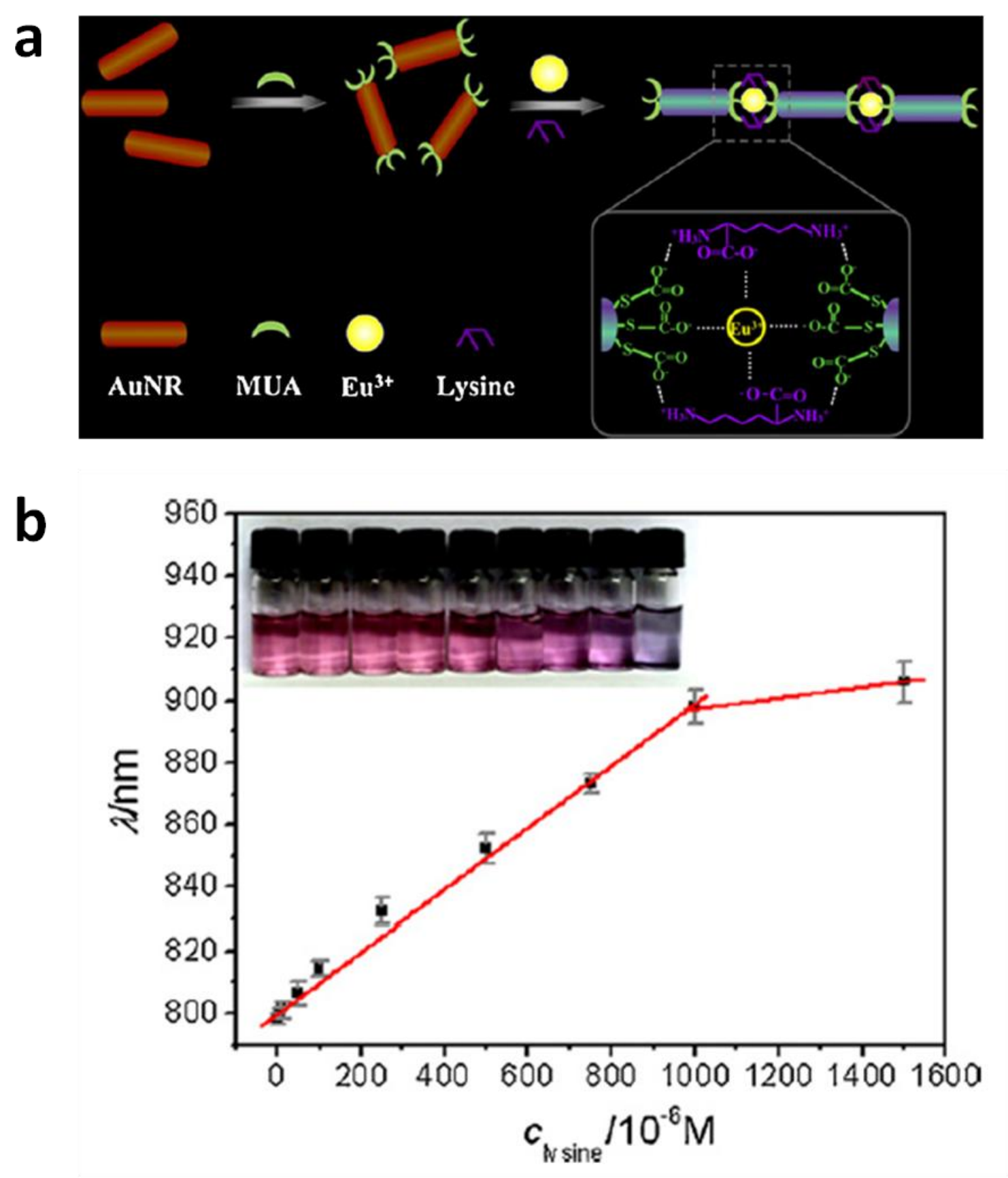

Figure 11: a) Schematic illustration of the assembly of Au nanorods in an end-to-end fashion mediated by $\mathrm{Eu}^{3+}$ and lysine. b) the assembly-based lysine recognition system undergoes a LSPR peak shift that is approximately linear with respect to the lysine concentration. Reprinted with permission from [133]. Copyright 2012 Elsevier.

Besides chemical linkers, biological linkers can also be used to enable detection of biomolecules. Au nanorods modified on the ends or sides using complementary microcystin 
(MC-LR) antibody and antigen were used for fast detection of MC-LR, an environmental toxin. The presence of MC-LR triggers end-to-end or side-to-side nanorod assembly and results in the enhancement of the longitudinal LSPR absorption. It was determined that end-to-end nanorod assemblies had markedly improved detection sensitivity and range than side-to-side assembly [138]. Similarly, ovalbumin (OVA) - antigen-modified and antibody-modified Au nanorods competed with the target analyte, Gentamicin (GM). This resulted in GM concentration dependent side-by-side nanorod assembly and UV-Vis absorption, which could be used to detect $\mathrm{GM}$ in the range of $0.1-20 \mathrm{ng} / \mathrm{mL}$ [132]. Au nanorods modified with specific DNA sequences that hybridize to the target DNA have been designed for DNA detection. This technique has been applied towards detection of $\mathrm{C}$. trachomatis pathogen, which triggers the aggregation of the nanorods and leads to the broadening and red shifting of the longitudinal LSPR [137].

\section{Surface Enhanced Raman Scattering}

The plasmonic coupling in nanorod assemblies also creates "hot spots" of strong local enhancement of the electromagnetic field. This phenomenon is widely used for surface enhanced Raman scattering (SERS), which is another powerful method of chemical and biological sensing [128]. When a probing electromagnetic beam is incident on the hotspot, the beam intensity is intensified by a power of 2 due to plasmonic enhancement. When target molecules in proximity to these hot spots are subsequently excited, the resulting SERS output signal can be amplified by another power of 2 , for a total signal amplification of an order of up to $10^{9}$ or more in comparison to substrates without plasmonic enhancement effects [141, 142].

Nanorods assembled on a flat substrate represent the most common configuration of nanorod-based SERS, because of its relative ease of characterization and compatibility with device scale processing and fabrication. Dong et al fabricated multilayered Au 
nanorod/polyelectrolyte nanostructures on the transparent electrode indium tin oxide (ITO) substrates using layer-by-layer assembly, utilizing strong electrostatic interactions between nanorods and polyelectrolyte to stabilize the structure. It was demonstrated that the multilayered nanorod structure could be used as a SERS substrate for probing 4-aminothiophenol (4-ATP). However, the nanorod ordering within each layer was poor, thus limiting the number of active SERS sites and resulting in only $60 \%$ of fabricated films demonstrating SERS enhancement [143]. Nogami et al achieved side-to-side and end-to-end assembly of Au nanorods on glass substrates by controlling CTAB concentration, and demonstrated the SERS detection of Rhodamin-6G molecules [144].

For practical and reproducible applications of nanorod assemblies for SERS, the nanorod assembly must be precisely defined over large areas and the fabrication technique must be cost effective and readily scalable. Specifically, vertical arrays of noble metal nanorods, used as SERS substrates, can achieve the highest sensitivities for detection due to accumulation of the plasmonic field at the tips of the nanorods [145]. To this end, various techniques have been developed to fabricate SERS substrates composed of strongly coupled vertical nanorod arrays. Several methods involve seeded nanorod growth, in which spherical nanoparticles are assembled into ordered arrays on substrates and act as seeds for the growth of vertical nanorods via a subsequent deposition step [146-149]. Nanorod arrays can also be fabricated using templated growth, in which a sacrificial template, such as anodic aluminum oxide (AAO) membrane, is situated on top of a substrate and the membrane pores are filled by a noble metal material. The template is subsequently removed to complete the fabrication of the vertically aligned nanorod arrays $[150,151]$. Although these fabrication methods do not strictly involve nanorod selfassembly, they display extremely high SERS signal enhancement factors and low limits of 
detection, and thus demonstrate the potential of vertically assembled nanorod arrays. Controlled drying has also been extensively studied for the large area, post synthesis assembly of noble metal nanorods into well-ordered vertical arrays as active SERS substrates. The orientation of the nanorods can be controlled via capillary forces [152], lithographic templating [153-155], ligand mediation [156-158], or application of external fields [89].

Besides flat SERS substrates, other nanorod configurations can also be used for SERS detection. Liz-Marzán et al used controlled drying to fabricate highly ordered Au nanorod supercrystals with plasmonic antennae enhancement of electrical field to enable real time direct detection of prions in blood with concentrations as low as $10^{-10} \mathrm{M}[159,160]$ (Figure 12). Controlled aggregation of nanorods into clusters and dimers have been used to create plasmonic hotspots for benzoyl peroxide [135] and Oxazine 725 reporter molecules [161]. Gold nanorods conjugated to anti-epidermal growth factor receptor (anti-EGFR) antibodies can be triggered by human oral cancer cells to assemble and align, resulting in enhanced, sharp, and polarized SERS signals [162]. Finally, electrospun polymer fibers containing nanorods oriented parallel to the fiber long axis have been used as flexible free-standing SERS substrates [163, 164]. 

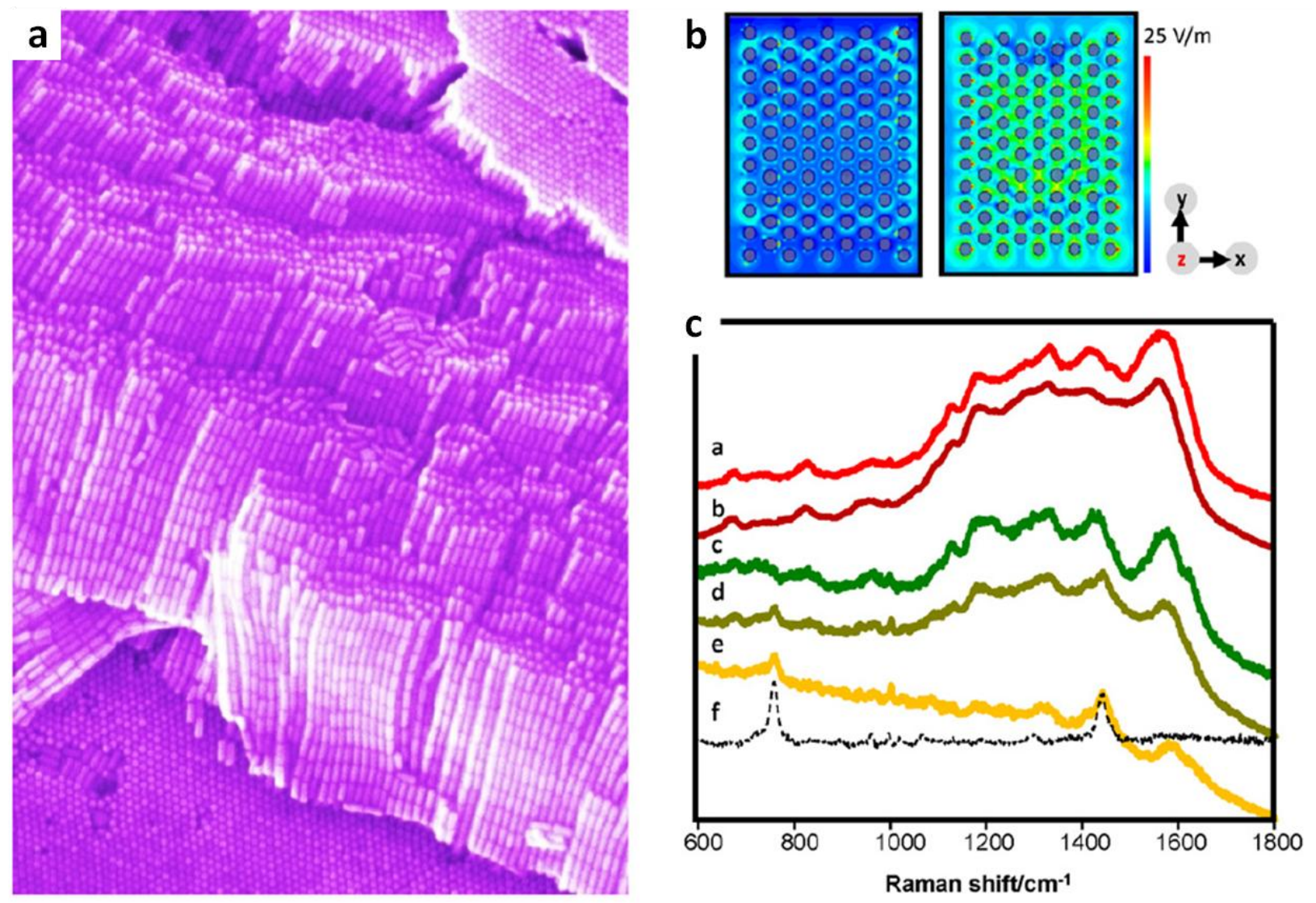

Figure 12: a) SEM image of a nanorod supercrystal-based SERS substrate. b) Electric field enhancement maps for single layer (left) and stack of three layers (right) of nanorods show that stacking of nanorod layers further enhance the strength of plasmonic "hotspots". c) SERS spectra of (from top to bottom): natural human blood, human blood spiked with prions, nature human plasma, human plasma spiked with prions, SERS spectrum of the spiked plasma after spectral subtraction of the matrix (human plasma), and SERS spectrum of the prion. Reprinted with permission from [160]. Copyright 2012 Elsevier.

\section{Circular Dichroism}

Recently, chiral assemblies of noble metal nanorods have garnered significant research interest due to their unique optical properties and potential for the sensing of chiral molecules. Liz-Marzán et al predicted large circular dichroism (CD) in chiral nanorod dimers due to asymmetric scattering and retardation of incident light as it interacts with the coupled electromagnetic field between the nanorods [165]. In practice, chiral nanorod assemblies can be 
achieved via templating methods. Chiral lipid [166], polymer fiber [167], and DNA [168] templates have been successfully employed to fabricate chiral nanorod superstructures with strong CD signal. Kotov et al used the hybridization of chiral DNA linkers to create chiral gold nanorod dimers [169], and demonstrated that by employing different reactant DNA templates and monitoring its effect on the resultant CD signal, attomolar concentrations of DNA molecules can be detected [170]. This chiroplasmonic method may be particularly useful for biological analytes larger than $2-5 \mathrm{~nm}$, which are difficult to detect by SERS. While chiral nanorod assemblies are promising for sensing applications, more studies are necessary to firmly establish the fundamental understanding of chiral templated assembly.

\section{Photovoltaics}

Semiconductor nanomaterials have found applications in photovoltaics, especially in organic/inorganic hybrid solar cells, because they provide high carrier transport, low trap density, and ease of bandgap tuning to improve charge separation and charge transport compared to organic solar cells [171]. Although many implementations of hybrid photovoltaics involve spherical semiconductor nanoparticles, charge transport in these devices are hindered by the presence of structural traps in the form of incomplete pathways in the percolation network [172]. Alivisatos et al first observed that hybrid photovoltaic devices containing elongated $\mathrm{CdSe}$ nanocrystals blended with poly (3-hexylthiophene) (P3HT) had power conversion efficiencies that are almost an order of magnitude higher than previous spherical nanocrystal/polymer heterojunction photovoltaic devices, and speculated that the improvement is due to denser assembly of nanorods in the polymer matrix and reduced number of hops required for the transport of excitons to the electrodes [173]. Subsequent studies with devices containing high aspect ratio CdSe nanorods demonstrated that controlling nanorod length can indeed be used to 
increase the distance on which electrons are transported directly through the thin film device. Furthermore, the bandgap can be readily tuned by altering the nanorod radius to optimize the overlap between the absorption spectrum of the cell and the solar emission spectrum [171].

Control over the order and orientation of the nanorods is also important to optimize the charge transport and separation in photovoltaic devices. Millo et al demonstrated that $\mathrm{CdSe}$ nanorods end-to-end aligned via controlled evaporation and subsequently ligand exchanged with diamine linkers exhibited strong photoconductivity [174]. Buriak et al used electrostatic layerby-layer assembly to generate multilayer CdSe nanorod/polymer composite films with the formation of intimate nanorod/conducting polymer heterojunctions. However, the nanorods are aligned parallel to the electrode surface in this case, and hence resulted in low device conversion efficiency [175]. Ideally, the nanorods should be aligned perpendicular to the electrodes to maximize the carrier transport distance. To this end, Russell et al took advantage of the permanent electric dipole in CdSe nanorods and used an external electric field to vertically align CdSe nanorods embedded in a P3HT matrix into densely packed arrays that stand perpendicularly to the underlying substrate [176]. Alivisatos et al demonstrated that such orientational control of nanorods can also be achieved by controlled drying over device scale areas [44], and that heterojunctions can be reversibly created within individual nanorods with precise compositional depths via cation exchange [177] (Figure 13). More recently, Ryan et al achieved evaporation assisted assembly of vertical arrays of $\mathrm{Cu}_{2} \mathrm{ZnSnS}_{4}$ nanorods, which are low-cost and nontoxic alternatives to CdSe nanorods [178]. These research efforts hold tremendous promise in realizing the full potential of nanorods within photovoltaic devices. 


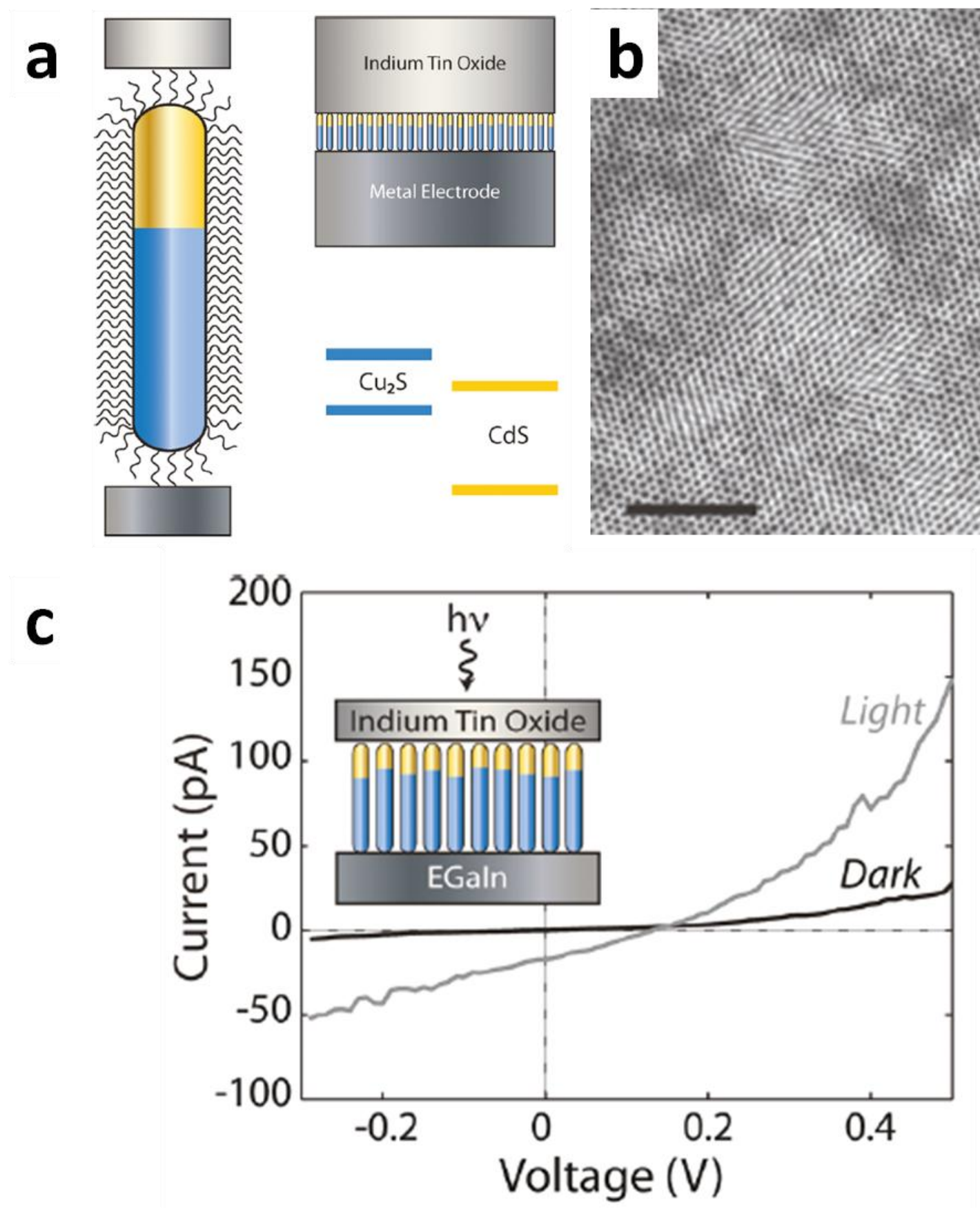

Figure 13: a) Schematic of self-assembled $\mathrm{Cu}_{2} \mathrm{~S} / \mathrm{CdS}$ nanorod array heterojunction photovoltaic devices. b) TEM image of aligned nanorod arrays via controlled drying over device scales (scale bar in b) is $100 \mathrm{~nm}$ ). c) Photovoltaic effect of the device demonstrated by current-voltage plot. Reprinted with permission from [177]. Copyright 2011 American Chemical Society. 


\section{Other Applications}

Besides the above highlighted applications, several other emerging applications also involve innovative uses of properties unique to nanorod assemblies. Colloidal $\mathrm{ZnO}$ nanorods are attractive materials for low-temperature and solution-processible thin film field effect transistors (TFTs). TFT devices made from nanorods via spin coating have been shown to have mobilities of $0.61 \mathrm{~cm}^{2} \mathrm{~V}-1 \mathrm{~s}^{-1}$ and on/off ratios of $3 \times 10^{5}$ after post-deposition growth, which is comparable to TFTs fabricated via traditional high temperature sputtering methods [179]. These nanorods can also be aligned parallel to the substrate and perpendicular to the electrodes by carefully tuning the nanorod ligands and drying kinetics. TFTs fabricated from these aligned nanorods exhibited mobilities of 1.2-1.4 $\mathrm{cm}^{2} \mathrm{~V}-1 \mathrm{~s}^{-1}$ and on/off ratios of $10^{6}-10^{7}$, a significant improvement compared to devices made from non-aligned nanorods [180].

Oriented assemblies of anisotropic nanocrystals also exhibit anisotropic optical properties. Weller et al first observed characteristic birefringence in highly aligned CdSe nanorod solids, which they attributed to the smectic ordering of nanorod layers within the solid [181]. Gacoin et al assembled parallel oriented $\mathrm{LaPO}_{4}$ nanorods using external shear via blade coating, and the resulting nanorod thin films exhibited remarkable birefringence $(\Delta n=0.13)$, which is promising for making thin film wave plates [182] (Figure 14). 

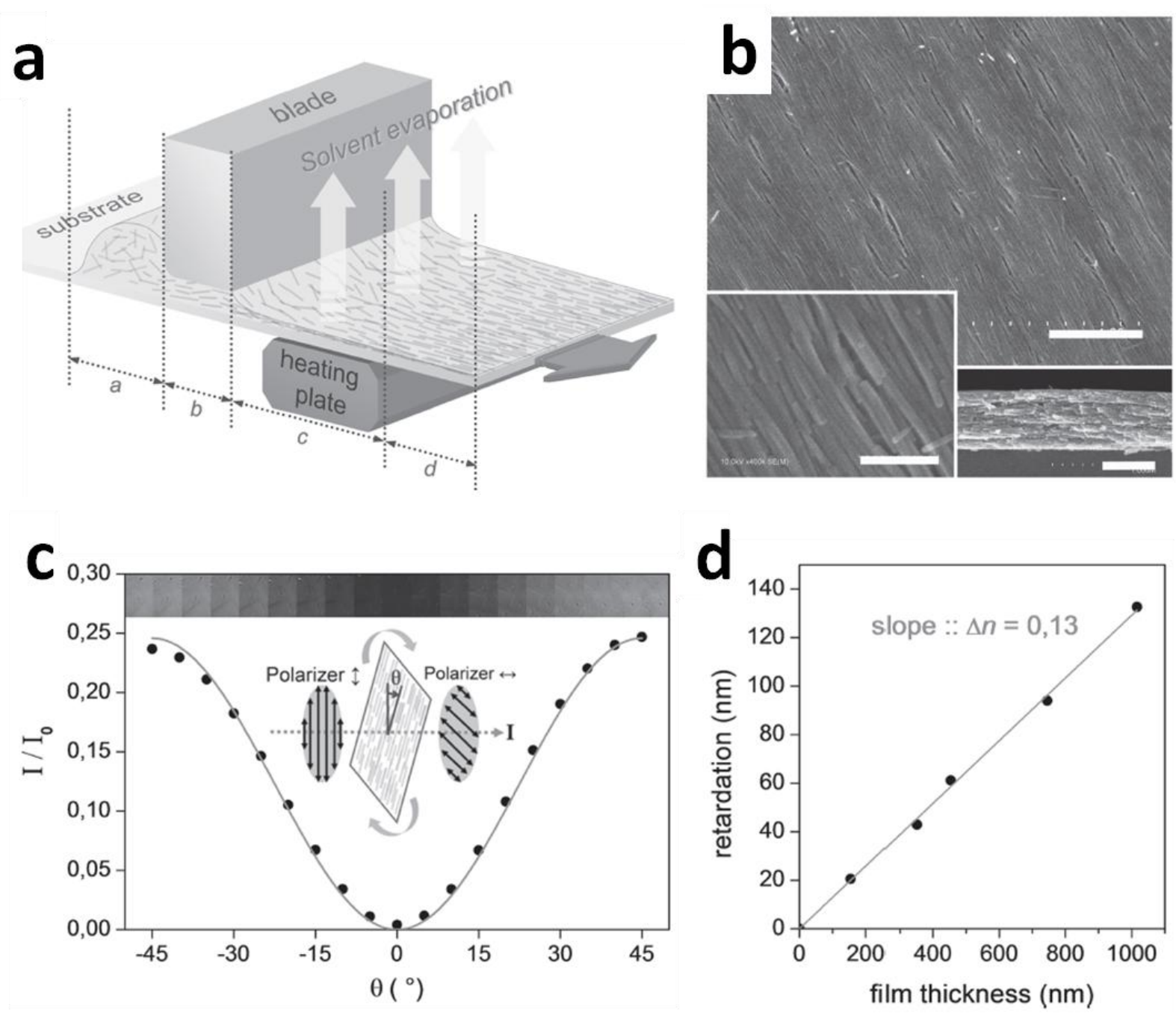

Figure 14: a) Schematic illustration of the fabrication of aligned $\mathrm{LaPO}_{4}$ nanorods via blade coating. b) SEM images of an aligned $\mathrm{LaPO}_{4}$ nanorod film surface and cross-section (scale bars are $500 \mathrm{~nm}$ for top and right panels, $100 \mathrm{~nm}$ for left panel). c) Normalized light intensity $(\lambda=$ $633 \mathrm{~nm}$ ) transmitted through the film inserted between cross polarizers as a function of sample azimuth angle $\theta$. d) Optical retardation as a function of film thickness, suggesting optical anisotropy of $\Delta n=0.13$. Reprinted with permission from [182]. Copyright 2013 Wiley.

The fluorescence of semiconductor nanorods can be harnessed collectively via selfassembly. In a study by Ryan et al, water dispersed semiconductor (CdS, CdSe, and CdTe) nanorod assemblies are formed by phase transfer of the nanorods from organic to aqueous solution using pluronic triblock copolymers. The encapsulated nanorods close pack in the form of discs in the hydrophobic core of the micelles. The order and axial alignment of the assembly 
allows fluorescence intensity to be enhanced via collective emission. Furthermore, the micelles showed excellent cellular uptake and cell specific fluorescence at low laser excitation intensities, thus demonstrating the potential of these nanorod assemblies as biomarkers [183].

\section{Conclusions and Perspectives}

Many pathways for the organization and alignment of anisotropic nanoscopic materials have been explored, each with their strengths and weaknesses. Non-directed methods are simple and quite elegant, but are rather limited in the morphologies they can access and can be very sensitive to factors such as solution concentration. Surface modification significantly expands the versatility of anisotropic nanomaterial assembly, but often requires challenging surface chemistry to modulate the nanomaterial properties. Applying external fields results in excellent orientational control of the nanomaterials, but has yet to demonstrate precise control over their spatial distribution. The use of prefabricated templates or a co-assembling polymer matrix can control both orientation and distribution, but require the potentially difficult fabrication of a template or control of the orientation of the co-assembling polymer, respectively. Therefore, there exist ample opportunities to develop improved assembly strategies to readily and simultaneously achieve orientational and positional control over the anisotropic nanomaterials over arbitrarily large volumes or areas.

Furthermore, it must be noted that nanostructures obtained via self-assembly approaches may not necessarily be in thermodynamic equilibrium. A number of metastable phases could potentially exist in local energy minima in addition to the thermodynamically stable phase. Therefore, a thorough understanding of the full energy landscape of the assembly process is crucial to distinguish the stable and metastable end structures, and potentially access additional complex nanostructures. The complexity of the system will necessitate the use of both 
computational and experimental methods to study the thermodynamics and dynamics of the assembly processes.

These research endeavors are well worth the effort and resources, as ordered assemblies of nanorods have vast potential in technological applications such as sensing, energy harvesting and transport, and data storage as well as applications yet to be explored. Nanorod-based chemical and biological sensors have demonstrated remarkable target specificity and low limits of detection that surpass commercially available alternatives. Nanorod heterojunction photovoltaic cells and thin film transistors have shown promise as functional and processible devices. Magnetic storage devices based on assembled nanorods is an intriguing and developing field, as the magnetic properties of ordered nanorods and their implementation into magnetic storage devices remain to be systematically explored. In addition, other applications of anisotropic nanomaterial assemblies, such as plasmonic waveguides and mechanically enhanced functional nanocomposites, are just beginning to emerge. The processing and structure/property correlations of these material systems are interesting and technological significant topics that require further research.

Furthermore, the availability, cost and monodispersity of the nanorods remain bottlenecks for large scale manufacturing of many nanorod-based technological devices. Although excellent synthesis protocols exist for traditional metal and semiconductor nanomaterials, the ability to tune the composition in a controlled and cost-effective way is crucial. Finally, assembly strategies that can be readily implemented into existing manufacturing infrastructure will further increase the viability of anisotropic nanomaterial assemblies as next generation advanced functional materials.

\section{Acknowledgements}


This work was supported by the Department of Energy, Office of Basic Energy Science under Contract DE-AC02-05CH11231 through the 'Organic-inorganic Nanocomposites' program.

\section{References}

[1] F.A. Aldaye, A.L. Palmer, H.F. Sleiman, Science, 321 (2008) 1795-1799.

[2] A.C. Balazs, T. Emrick, T.P. Russell, Science, 314 (2006) 1107-1110.

[3] A.K. Boal, F. Ilhan, J.E. DeRouchey, T. Thurn-Albrecht, T.P. Russell, V.M. Rotello, Nature, 404 (2000) 746-748.

[4] S.C. Glotzer, M.J. Solomon, Nat Mater, 6 (2007) 557-562.

[5] W.U. Huynh, J.J. Dittmer, A.P. Alivisatos, Science, 295 (2002) 2425-2427.

[6] M.R. Bockstaller, R.A. Mickiewicz, E.L. Thomas, Adv. Mater., 17 (2005) 1331-1349.

[7] Q. Liu, Y. Cui, D. Gardner, X. Li, S. He, I.I. Smalyukh, Nano Lett., 10 (2010) 1347-1353.

[8] G.A. Buxton, A.C. Balazs, Mol. Simulat., 30 (2004) 249-257.

[9] E. Auyeung, R.J. Macfarlane, C.H.J. Choi, J.I. Cutler, C.A. Mirkin, Adv. Mater., 24 (2012) 5181-5186.

[10] E. Dujardin, S. Mann, L.-B. Hsin, C.R.C. Wang, Chem. Commun., (2001) 1264-1265.

[11] M.R. Jones, R.J. Macfarlane, B. Lee, J. Zhang, K.L. Young, A.J. Senesi, C.A. Mirkin, Nat. Mater., 9 (2010) 913-917.

[12] D. Fava, Z. Nie, M.A. Winnik, E. Kumacheva, Adv. Mater., 20 (2008) 4318-4322.

[13] M.A. Horsch, M.H. Lamm, S.C. Glotzer, Nano Lett., 3 (2003) 1341-1346.

[14] M. Rycenga, J.M. McLellan, Y. Xia, Adv. Mater., 20 (2008) 2416-2420.

[15] M. Sethi, G. Joung, M.R. Knecht, Langmuir, 25 (2009) 1572-1581.

[16] D.A. Walker, V.K. Gupta, Nanotechnology, 19 (2008) 435603.

[17] C.J. Murphy, C.J. Orendorff, Adv. Mater., 17 (2005) 2173-2177.

[18] K.M. Ryan, A. Mastroianni, K.A. Stancil, H. Liu, A.P. Alivisatos, Nano Lett., 6 (2006) 1479-1482. 
[19] B.W. Smith, Z. Benes, D.E. Luzzi, J.E. Fischer, D.A. Walters, M.J. Casavant, J. Schmidt, R.E. Smalley, Appl. Phys. Lett., 77 (2000) 663.

[20] P. Wang, L. Zhang, Y. Xia, L. Tong, X. Xu, Y. Ying, Nano Lett., (2012) 3145-3150.

[21] R.D. Deshmukh, Y. Liu, R.J. Composto, Nano Lett., 7 (2007) 3662-3668.

[22] M.J.A. Hore, R.J. Composto, ACS Nano, 4 (2010) 6941-6949.

[23] L. Li, C. Miesch, P.K. Sudeep, A.C. Balazs, T. Emrick, T.P. Russell, R.C. Hayward, Nano Lett., 11 (2011) 1997-2003.

[24] E. Ploshnik, A. Salant, U. Banin, R. Shenhar, Adv. Mater., 22 (2010) 2774-2779.

[25] J. Kao, K. Thorkelsson, P. Bai, B.J. Rancatore, T. Xu, Chem. Soc. Rev., 42 (2013) 26542678.

[26] M.J.A. Hore, R.J. Composto, Macromolecules, 47 (2014) 875-887.

[27] D. Wang, M.J.A. Hore, X. Ye, C. Zheng, C.B. Murray, R.J. Composto, Soft Matter, 10 (2014) 3404-3413.

[28] D. Baranov, A. Fiore, M. van Huis, C. Giannini, A. Falqui, U. Lafont, H. Zandbergen, M. Zanella, R. Cingolani, L. Manna, Nano Lett., 10 (2010) 743-749.

[29] S.V. Savenko, M. Dijkstra, J. Chem. Phys., 124 (2006) 234902.

[30] N. Denkov, O. Velev, P. Kralchevski, I. Ivanov, H. Yoshimura, K. Nagayama, Langmuir, 8 (1992) 3183-3190.

[31] T.D. Clark, J. Tien, D.C. Duffy, K.E. Paul, G.M. Whitesides, J. Am. Chem. Soc., 123 (2001) 7677-7682.

[32] A.V. Titov, P. Král, Nano Lett., 8 (2008) 3605-3612.

[33] P.G. Bolhuis, A. Stroobants, D. Frenkel, H.N.W. Lekkerkerker, J. Chem. Phys., 107 (1997) 1551-1564.

[34] L.-S. Li, J. Walda, L. Manna, A.P. Alivisatos, Nano Lett., 2 (2002) 557-560.

[35] N.R. Jana, L.A. Gearheart, S.O. Obare, C.J. Johnson, K.J. Edler, S. Mann, C.J. Murphy, J. Mater. Chem., 12 (2002) 2909-2912.

[36] C.-C. Kang, C.-W. Lai, H.-C. Peng, J.-J. Shyue, P.-T. Chou, ACS Nano, 2 (2008) 750-756. 
[37] M. Zanella, G. Bertoni, I.R. Franchini, R. Brescia, D. Baranov, L. Manna, Chem. Commun., 47 (2011) 203-205.

[38] B. Nikoobakht, Z.L. Wang, M.A. El-Sayed, J. Phys. Chem. B, 104 (2000) 8635-8640.

[39] L.-S.L.-S. Li, A.P. Alivisatos, Adv. Mater., 15 (2003) 408-411.

[40] A. Ghezelbash, B. Koo, B.A. Korgel, Nano Lett., 6 (2006) 1832-1836.

[41] B.P. Khanal, E.R. Zubarev, Angew. Chem. Int. Edit., 46 (2007) 2195-2198.

[42] Z.-C. Xu, C.-M. Shen, C.-W. Xiao, T.-Z. Yang, S.-T. Chen, H.-L. Li, H.-J. Gao, Chem. Phys. Lett., 432 (2006) 222-225.

[43] C. Querner, M.D. Fischbein, P.A. Heiney, M. Drndić, Adv. Mater., 20 (2008) 2308-2314.

[44] J.L. Baker, A. Widmer-Cooper, M.F. Toney, P.L. Geissler, A.P. Alivisatos, Nano Lett., 10 (2010) 195-201.

[45] R. Blaak, B.M. Mulder, D. Frenkel, J. Chem. Phys., 120 (2004) 5486-5492.

[46] B.S. John, A. Stroock, F.a. Escobedo, J. Chem. Phys., 120 (2004) 9383-9389.

[47] U. Agarwal, F.A. Escobedo, Nat. Mater., 10 (2011) 230-235.

[48] P.F. Damasceno, M. Engel, S.C. Glotzer, Science, 337 (2012) 453-457.

[49] E.V. Shevchenko, D.V. Talapin, N.A. Kotov, S. O'Brien, C.B. Murray, Nature, 439 (2006) 55-59.

[50] X. Ye, J. Chen, M. Engel, J.A. Millan, W. Li, L. Qi, G. Xing, J.E. Collins, C.R. Kagan, J. Li, S.C. Glotzer, C.B. Murray, Nat. Chem., 5 (2013) 466-473.

[51] W. Qi, J.d. Graaf, F. Qiao, S. Marras, L. Manna, M. Dijkstra, Nano Lett., 12 (2012) 52995303.

[52] M.P. Arciniegas, M.R. Kim, J. De Graaf, R. Brescia, S. Marras, K. Miszta, M. Dijkstra, R. van Roij, L. Manna, Nano Lett., 14 (2014) 1056-1063.

[53] K. Miszta, J. de Graaf, G. Bertoni, D. Dorfs, R. Brescia, S. Marras, L. Ceseracciu, R. Cingolani, R. van Roij, M. Dijkstra, L. Manna, Nat. Mater., 10 (2011) 872-876.

[54] T. Ming, X. Kou, H. Chen, T. Wang, H.-L. Tam, K.-W. Cheah, J.-Y. Chen, J. Wang, Angew. Chem. Int. Edit., 47 (2008) 9685-9690. 
[55] J. Henzie, M. Grünwald, A. Widmer-Cooper, P.L. Geissler, P. Yang, Nat. Mater., 11 (2011) 131-137.

[56] N. Yanai, S. Granick, Angew. Chem. Int. Edit., 51 (2012) 5638-5641.

[57] J.C.P. Gabriel, P. Davidson, Mineral liquid crystals from self-assembly of anisotropic nanosystems, in: M. Antonietti (Ed.) Colloid Chem. 12003, pp. 119-172.

[58] Z. Quan, J. Fang, Nano Today, 5 (2010) 390-411.

[59] P.R. Sajanlal, T.S. Sreeprasad, A.K. Samal, T. Pradeep, Nano Rev., 2 (2011).

[60] F. Dumestre, B. Chaudret, C. Amiens, M. Respaud, P. Fejes, P. Renaud, P. Zurcher, Angew. Chem. Int. Edit., 42 (2003) 5213-5216.

[61] D.V. Talapin, E.V. Shevchenko, C.B. Murray, A. Kornowski, S. Förster, H. Weller, J. Am. Chem. Soc., 126 (2004) 12984-12988.

[62] C. Knorowski, A. Travesset, J. Am. Chem. Soc., 136 (2014) 653-659.

[63] S. Vial, D. Nykypanchuk, K.G. Yager, A.V. Tkachenko, O. Gang, ACS Nano, 7 (2013) 5437-5445.

[64] W. Ni, R.A. Mosquera, J. Pérez-Juste, L.M. Liz-Marzán, J. Phys. Chem. Lett., 1 (2010) 1181-1185.

[65] R.C. Ferrier, H.-S. Lee, M.J.A. Hore, M. Caporizzo, D.M. Eckmann, R.J. Composto, Langmuir, 30 (2014) 1906-1914.

[66] K.G. Thomas, S. Barazzouk, B.I. Ipe, S.T.S. Joseph, P.V. Kamat, J. Phys. Chem. B, 108 (2004) 13066-13068.

[67] Z. Zhang, A.S. Keys, T. Chen, S.C. Glotzer, Langmuir, 21 (2005) 11547-11551.

[68] K.K. Caswell, J.N. Wilson, U.H.F. Bunz, C.J. Murphy, J. Am. Chem. Soc., 125 (2003) 13914-13915.

[69] A. Gole, C.J. Murphy, Langmuir, 21 (2005) 10756-10762.

[70] M.H. Zareie, X. Xu, M.B. Cortie, Small, 3 (2007) 139-145.

[71] P.R. Selvakannan, E. Dumas, F. Dumur, C. Péchoux, P. Beaunier, A. Etcheberry, F. Sécheresse, H. Remita, C.R. Mayer, J. Colloid Interf. Sci., 349 (2010) 93-97.

[72] D. Fava, M.A. Winnik, E. Kumacheva, Chem. Commun., (2009) 2571-2573.

[73] T.S. Sreeprasad, A.K. Samal, T. Pradeep, Langmuir, 24 (2008) 4589-4599. 
[74] H.-S. Park, A. Agarwal, N.A. Kotov, O.D. Lavrentovich, Langmuir, 24 (2008) 13833-13837.

[75] C.J. Orendorff, T.M. Alam, D.Y. Sasaki, B.C. Bunker, J.A. Voigt, ACS Nano, 3 (2009) 971-983.

[76] N. Zhao, K. Liu, J. Greener, Z. Nie, E. Kumacheva, Nano Lett., 9 (2009) 3077-3081.

[77] A. Salant, E. Amitay-Sadovsky, U. Banin, J. Am. Chem. Soc., 128 (2006) 10006-10007.

[78] A. Figuerola, I.R. Franchini, A. Fiore, R. Mastria, A. Falqui, G. Bertoni, S. Bals, G. Van Tendeloo, S. Kudera, R. Cingolani, L. Manna, Adv. Mater., 21 (2009) 550-554.

[79] M.A. Horsch, Z. Zhang, S.C. Glotzer, Phys. Rev. Lett., 95 (2005) 056105.

[80] T.D. Nguyen, S.C. Glotzer, Small, 5 (2009) 2092-2098.

[81] M.A. Horsch, Z. Zhang, S.C. Glotzer, Soft Matter, 6 (2010) 945-954.

[82] T.D. Nguyen, S.C. Glotzer, ACS Nano, 4 (2010) 2585-2594.

[83] K.D. Hermanson, S.O. Lumsdon, J.P. Williams, E.W. Kaler, O.D. Velev, Science, 294 (2001) 1082-1086.

[84] O.D. Velev, K.H. Bhatt, Soft Matter, 2 (2006) 738-750.

[85] E. Rossitto, O. Lecarme, L. Latu-Romain, K. Berton, T. Pinedo-Rivera, T. Jiu, P. Reiss, D. Peyrade, Microelectron. Eng., 86 (2009) 828-831.

[86] S. Gupta, Q. Zhang, T. Emrick, T.P. Russell, Nano Lett., 6 (2006) 2066-2069.

[87] S. Singamaneni, V.N. Bliznyuk, C. Binek, E.Y. Tsymbal, J. Mater. Chem., 21 (2011) 16819.

[88] Y. Bao, H. Fong, C. Jiang, J. Phys. Chem. C, 117 (2013) 21490-21497.

[89] S. Umadevi, X. Feng, T. Hegmann, Adv. Funct. Mater., 23 (2013) 1393-1403.

[90] Q. Liu, J. Tang, Y. Zhang, A. Martinez, S. Wang, S. He, T.J. White, I.I. Smalyukh, Phys. Rev. E, 89 (2014) 052505.

[91] M. Campbell, Q. Liu, A. Sanders, J. Evans, I. Smalyukh, Materials, 7 (2014) 3021-3033.

[92] J.S. Evans, C.N. Beier, I.I. Smalyukh, J. Appl. Phys., 110 (2011) 033535.

[93] Q. Liu, B. Senyuk, J. Tang, T. Lee, J. Qian, S. He, I. Smalyukh, Phys. Rev. Lett., 109 (2012) 088301. 
[94] Q. Liu, M.G. Campbell, J.S. Evans, I.I. Smalyukh, Adv. Mater., 26 (2014) 7178-7184.

[95] F. Kim, S. Kwan, J. Akana, P. Yang, J. Am. Chem. Soc., 123 (2001) 4360-4361.

[96] J. He, Q. Zhang, S. Gupta, T. Emrick, T.P. Russell, P. Thiyagarajan, Small, 3 (2007) 12141217.

[97] M.D. Goodman, L. Zhao, K.a. Derocher, J. Wang, S.K. Mallapragada, Z. Lin, ACS Nano, 4 (2010) 2043-2050.

[98] M.a. Correa-Duarte, J. Pérez-Juste, A. Sánchez-Iglesias, M. Giersig, L.M. Liz-Marzán, Angew. Chem. Int. Edit., 44 (2005) 4375-4378.

[99] Y. Cui, M.T. Björk, J.A. Liddle, C. Sönnichsen, B. Boussert, A.P. Alivisatos, Nano Lett., 4 (2004) 1093-1098.

[100] S.L. Teich-McGoldrick, M. Bellanger, M. Caussanel, L. Tsetseris, S.T. Pantelides, S.C. Glotzer, R.D. Schrimpf, Nano Lett., 9 (2009) 3683-3688.

[101] D. Nepal, M.S. Onses, K. Park, M. Jespersen, C.J. Thode, P.F. Nealey, R.a. Vaia, ACS Nano, (2012) 5693-5701.

[102] M.J.A. Hore, A.L. Frischknecht, R.J. Composto, ACS Macro Lett., 1 (2012) 115-121.

[103] D. Wang, M.J.A. Hore, X. Ye, C. Zheng, C.B. Murray, R.J. Composto, Soft Matter, (2014) 3404-3413.

[104] W. Li, B. Dong, L.-T. Yan, Macromolecules, 46 (2013) 7465-7476.

[105] F.S. Bates, M.A. Hillmyer, T.P. Lodge, C.M. Bates, K.T. Delaney, G.H. Fredrickson, Science, 336 (2012) 434-440.

[106] K. Chen, Y.-Q. Ma, J. Chem. Phys., 116 (2002) 7783-7786.

[107] Q.-Y. Tang, Y.-q. Ma, J. Phys. Chem. B, 113 (2009) 10117-10120.

[108] L. He, L. Zhang, H. Chen, H. Liang, Polymer, 50 (2009) 3403-3410.

[109] L. He, L. Zhang, H. Liang, Polymer, 51 (2010) 3303-3314.

[110] A. Halevi, S. Halivni, M. Oded, A.H.E. Muller, U. Banin, R. Shenhar, Macromolecules, 47 (2014) 3022-3032.

[111] Z. Shou, G.A. Buxton, A.C. Balazs, Compos. Interface., 10 (2003) 343-368.

[112] Y. Liu, O. Kuksenok, A.C. Balazs, Langmuir, 29 (2013) 750-760. 
[113] Y.V. Kudryavtsev, E.N. Govorun, A.D. Litmanovich, H.R. Fischer, Macromol. Theor. Simul., 13 (2004) 392-399.

[114] L.-T. Yan, N. Popp, S.-K. Ghosh, A. Böker, ACS Nano, 4 (2010) 913-920.

[115] H. Chen, D.F. Schmidt, M. Pitsikalis, N. Hadjichristidis, Y. Zhang, U. Wiesner, E.P. Giannelis, J. Polym. Sci. Pol. Phys., 41 (2003) 3264-3271.

[116] M. Laus, O. Francescangeli, F. Sandrolini, J. Mater. Res., 12 (2011) 3134-3139.

[117] C.A. Mitchell, R. Krishnamoorti, J. Polym. Sci. Pol. Phys., 40 (2002) 1434-1443.

[118] W. Li, P. Zhang, M. Dai, J. He, T. Babu, Y.-L. Xu, R. Deng, R. Liang, M.-H. Lu, Z. Nie, J. Zhu, Macromolecules, 46 (2013) 2241-2248.

[119] K. Thorkelsson, A.J. Mastroianni, P. Ercius, T. Xu, Nano Lett., 12 (2012) 498-504.

[120] K. Thorkelsson, J.H. Nelson, A.P. Alivisatos, T. Xu, Nano Lett., 13 (2013) 4908-4913.

[121] H. Chen, L. Shao, Q. Li, J. Wang, Chem. Soc. Rev., 42 (2013) 2679-2724.

[122] J. Perez-Juste, I. Pastoriza-Santos, L.M. Liz-Marzan, P. Mulvaney, Coordin. Chem. Rev., 249 (2005) 1870-1901.

[123] G.C. Yi, C.R. Wang, W.I. Park, Semicond. Sci. Tech., 20 (2005) S22-S34.

[124] L.M. Liz-Marzán, Langmuir, 22 (2005) 32-41.

[125] X. Huang, S. Neretina, M.A. El-Sayed, Adv. Mater., 21 (2009) 4880-4910.

[126] R. Gans, Ann. Phys., 352 (1915) 270-284.

[127] P.K. Jain, S. Eustis, M.A. El-Sayed, J. Phys. Chem. B, 110 (2006) 18243-18253.

[128] K. Liu, N. Zhao, E. Kumacheva, Chem. Soc. Rev., 40 (2011) 656-671.

[129] L. Vigderman, B.P. Khanal, E.R. Zubarev, Adv. Mater., 24 (2012) 4811-4841.

[130] M.K. Gupta, T. König, R. Near, D. Nepal, L.F. Drummy, S. Biswas, S. Naik, R.A. Vaia, M.A. El-Sayed, V.V. Tsukruk, Small, 9 (2013) 2979-2990.

[131] T. Placido, G. Aragay, J. Pons, R. Comparelli, M.L. Curri, A. Merkoci, ACS Appl. Mater. Interfaces, 5 (2013) 1084-1092.

[132] Y. Zhu, C. Qu, H. Kuang, L. Xu, L. Liu, Y. Hua, L. Wang, C. Xu, Biosens. Bioelectron., 26 (2012) 4387-4392. 
[133] J. Wang, P. Zhang, C.M. Li, Y.F. Li, C.Z. Huang, Biosens. Bioelectron., 34 (2012) 197201.

[134] T.S. Sreeprasad, T. Pradeep, Langmuir, 27 (2011) 3381-3390.

[135] Z. Jiang, G. Wen, Y. Luo, X. Zhang, Q. Liu, A. Liang, Sci. Rep., 4 (2014) 5323.

[136] X. Fu, L. Chen, J. Li, M. Lin, H. You, W. Wang, Biosens. Bioelectron., 34 (2012) 227-231.

[137] H.J. Parab, C. Jung, J.-H. Lee, H.G. Park, Biosens. Bioelectron., 26 (2010) 667-673.

[138] L. Wang, Y. Zhu, L. Xu, W. Chen, H. Kuang, L. Liu, A. Agarwal, C. Xu, N.A. Kotov, Agnew. Chem. Int. Edit., 49 (2010) 5472-5475.

[139] H. Nakashima, K. Furukawa, Y. Kashimura, K. Torimitsu, Chem. Commun., (2007) 10801082.

[140] N. Xiao, C. Yu, Anal. Chem., 82 (2010) 3659-3663.

[141] J. Kneipp, H. Kneipp, K. Kneipp, Chem. Soc. Rev., 37 (2008) 1052-1060.

[142] K. Kneipp, H. Kneipp, J. Kneipp, Accounts Chem. Res., 39 (2006) 443-450.

[143] X.G. Hu, W.L. Cheng, T. Wang, Y.L. Wang, E.K. Wang, S.J. Dong, J. Phys. Chem. B, 109 (2005) 19385-19389.

[144] G. Kawamura, Y. Yang, M. Nogami, Appl. Phys. Lett., 90 (2007) 261908.

[145] W. Wei, K. Chen, G. Ge, Adv. Mater., 25 (2013) 3863-3868.

[146] L.A. Dick, A.D. McFarland, C.L. Haynes, R.P. Van Duyne, J. Phys. Chem. B, 106 (2002) 853-860.

[147] N.G. Greeneltch, M.G. Blaber, G.C. Schatz, R.P. Van Duyne, J. Phys. Chem. C, 117 (2013) 2554-2558.

[148] M. Litorja, C.L. Haynes, A.J. Haes, T.R. Jensen, R.P. Van Duyne, J. Phys. Chem. B, 105 (2001) 6907-6915.

[149] B. Peng, G. Li, D. Li, S. Dodson, Q. Zhang, J. Zhang, Y.H. Lee, H.V. Demir, X.Y. Ling, Q. Xiong, ACS Nano, 7 (2013) 5993-6000.

[150] Q. Liao, C. Mu, D.-S. Xu, X.-C. Ai, J.-N. Yao, J.-P. Zhang, Langmuir, 25 (2009) 47084714.

[151] Z. Huang, G. Meng, Q. Huang, B. Chen, C. Zhu, Z. Zhang, J. Raman. Spectrosc., 44 (2013) 240-246. 
[152] T. Ming, X. Kou, H. Chen, T. Wang, H.-L. Tam, K.-W. Cheah, J.-Y. Chen, J. Wang, Agnew. Chem. Int. Edit., 47 (2008) 9685-9690.

[153] C. Hamon, M. Postic, E. Mazari, T. Bizien, C. Dupuis, P. Even-Hernandez, A. Jimenez, L. Courbin, C. Gosse, F. Artzner, V. Marchi-Artzner, ACS Nano, 6 (2012) 4137-4146.

[154] T. Thibaut, Y. Zheng, S.H. Ng, S. Mudie, M. Altissimo, U. Bach, Agnew. Chem. Int. Edit., 51 (2012) 8732-8735.

[155] J. Xiao, Z. Li, X. Ye, Y. Ma, L. Qi, Nanoscale, 6 (2014) 996-1004.

[156] S. Gomez-Grana, J. Perez-Juste, R.A. Alvarez-Puebla, A. Guerrero-Martinez, L.M. LizMarzan, Adv. Opt. Mater., 1 (2013) 477-481.

[157] A. Guerrero-Martinez, J. Perez-Juste, E. Carbo-Argibay, G. Tardajos, L.M. Liz-Marzan, Agnew. Chem. Int. Edit., 48 (2009) 9484-9488.

[158] C. Hamon, T. Bizien, F. Artzner, P. Even-Hernandez, V. Marchi, J. Colloid Interf. Sci., 424 (2014) 90-97.

[159] R.A. Alvarez-Puebla, A. Agarwal, P. Manna, B.P. Khanal, P. Aldeanueva-Potel, E. CarboArgibay, N. Pazos-Perez, L. Vigderman, E.R. Zubarev, N.A. Kotov, L.M. Liz-Marzan, P. Natl. Acad. Sci. USA, 108 (2011) 8157-8161.

[160] R.A. Alvarez-Puebla, E.R. Zubarev, N.A. Kotov, L.M. Liz-Marzan, Nano Today, 7 (2012) 6-9.

[161] A.F. Stewart, A. Lee, A. Ahmed, S. Ip, E. Kumacheva, G.C. Walker, ACS Nano, 8 (2014) 5462-5467.

[162] X. Huang, I.H. El-Sayed, W. Qian, M.A. El-Sayed, Nano Lett., 7 (2007) 1591-1597.

[163] Y. Bao, H. Fong, C. Jiang, J. Phys. Chem. C, 117 (2013) $21490-21497$.

[164] C.-L. Zhang, K.-P. Lv, H.-P. Cong, S.-H. Yu, Small, 8 (2012) 648-653.

[165] B. Auguie, J. Lorenzo Alonso-Gomez, A. Guerrero-Martinez, L.M. Liz-Marzan, J. Phys. Chem. Lett., 2 (2011) 846-851.

[166] R.-Y. Wang, H. Wang, X. Wu, Y. Ji, P. Wang, Y. Qu, T.-S. Chung, Soft Matter, 7 (2011) 8370-8375.

[167] A. Guerrero-Martinez, B. Auguie, J. Lorenzo Alonso-Gomez, Z. Dzolic, S. Gomez-Grana, M. Zinic, M. Magdalena Cid, L.M. Liz-Marzan, Agnew. Chem. Int. Edit., 50 (2011) 5499-5503. 
[168] Z. Li, Z. Zhu, W. Liu, Y. Zhou, B. Han, Y. Gao, Z. Tang, J. Am. Chem. Soc., 134 (2012) 3322-3325.

[169] W. Ma, H. Kuang, L. Wang, L. Xu, W.-S. Chang, H. Zhang, M. Sun, Y. Zhu, Y. Zhao, L. Liu, C. Xu, S. Link, N.A. Kotov, Sci. Rep., 3 (2013) 1934.

[170] W. Ma, H. Kuang, L. Xu, L. Ding, C. Xu, L. Wang, N.A. Kotov, Nat. Commun., 4 (2013) 2689.

[171] W.U. Huynh, J.J. Dittmer, A.P. Alivisatos, Science, 295 (2002) 2425-2427.

[172] J.J. Dittmer, E.A. Marseglia, R.H. Friend, Adv. Mater., 12 (2000) 1270-1274.

[173] W.U. Huynh, X.G. Peng, A.P. Alivisatos, Adv. Mater., 11 (1999) 923-927.

[174] D. Steiner, D. Azulay, A. Aharoni, A. Salant, U. Banin, O. Millo, Phys. Rev. B, 80 (2009) 195308.

[175] S.A. McClure, B.J. Worfolk, D.A. Rider, R.T. Tucker, J.A.M. Fordyce, M.D. Fleischauer, K.D. Harris, M.J. Brett, J.M. Buriak, ACS Appl. Mater. Interfaces, 2 (2010) 219-229.

[176] S. Gupta, Q. Zhang, T. Emrick, T.P. Russell, Nano Lett., 6 (2006) 2066-2069.

[177] J.B. Rivest, S.L. Swisher, L.-K. Fong, H. Zheng, A.P. Alivisatos, ACS Nano, 5 (2011) 3811-3816.

[178] A. Singh, H. Geaney, F. Laffir, K.M. Ryan, J. Am. Chem. Soc., 134 (2012) 2910-2913.

[179] B. Sun, H. Sirringhaus, Nano Lett., 5 (2005) 2408-2413.

[180] B. Sun, H. Sirringhaus, J. Am. Chem. Soc., 128 (2006) 16231-16237.

[181] D.V. Talapin, E.V. Shevchenko, C.B. Murray, A. Kornowski, S. Forster, H. Weller, J. Am. Chem. Soc., 126 (2004) 12984-12988.

[182] J. Kim, J. Peretti, K. Lahlil, J.-P. Boilot, T. Gacoin, Adv. Mater., 25 (2013) 3295-3300.

[183] A. Sanyal, T. Bala, S. Ahmed, A. Singh, A.V. Piterina, T.M. McGloughlin, F.R. Laffir, K.M. Ryan, J. Mater. Chem., 19 (2009) 8974-8981. 


\section{Vitae}

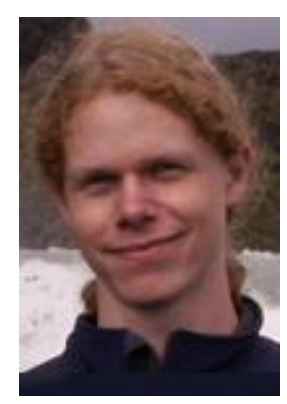

Kari Thorkelsson received his BS degrees in Materials Science and Engineering and Chemical Engineering in 2009 and a Ph.D. degree in Materials Science and Engineering in 2014 from the University of California, Berkeley. He is currently employed as a process engineer by Lam Research.

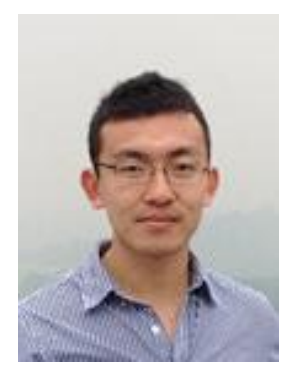

Peter Bai received his BS degree in Materials Science and Engineering from Cornell University in 2010. He received his MS degree in Materials Science and Engineering in 2011 from the University of California, Berkeley, where he researched nanoparticle assembly in block copolymer-based supramolecular thin films. Currently he is a PhD student in Materials Science and Engineering at the University of California, Berkeley, investigating the assembly of supramolecular nanocomposites in bulk, thin films, and under cylindrical confinement towards functional materials.

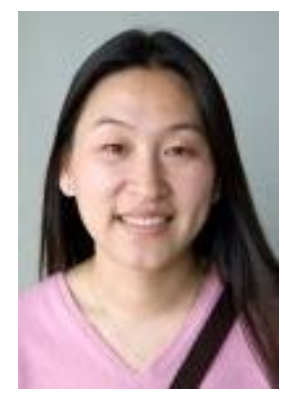


Ting Xu received an MS degree from Changchun Institute of Applied Chemistry, Chinese Academy of Science in 1999 and a PhD from the Department of Polymer Science and Engineering at the University of Massachusetts, Amherst in 2004. She was a joint postdoctoral fellow between the University of Pennsylvania and the Cold Neutrons for Biology and Technology Partnership Program at NIST. She is now an Associate Professor in the Department of Materials Science and Engineering and the Department of Chemistry at UC Berkeley, and a Faculty Scientist at Lawrence Berkeley National Laboratory. Her research efforts focus on fundamentally understanding the hierarchical self-assembly of complex systems toward functional hybrid materials. 


\section{Graphical Abstract}

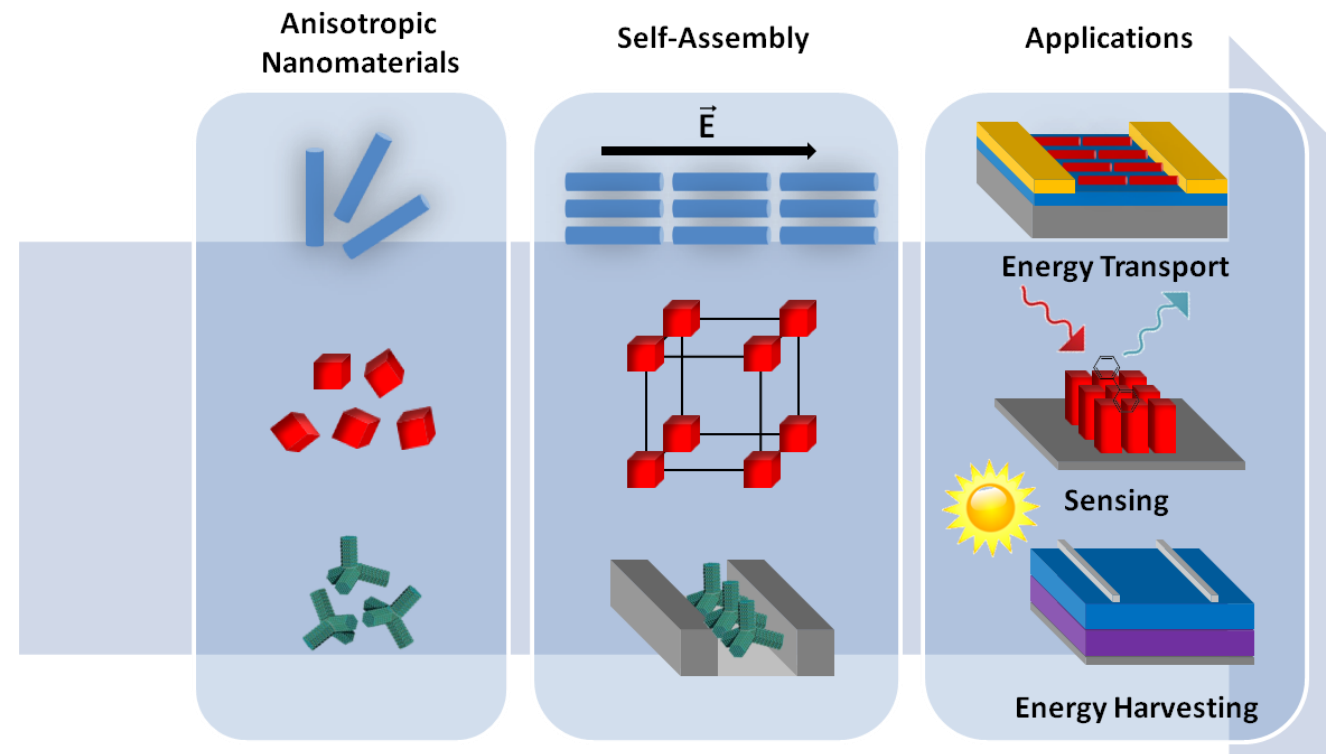

